\title{
Non-dispersive and weakly dispersive single- layer flow over an axisymmetric obstacle: the equivalent aerofoil formulation
}

\author{
J. G. ESLER, O. J. RUMP AND E. R. JOHNSON \\ Department of Mathematics, University College London, 25 Gower Street, London WC1E 6BT, UK \\ gavin@math.ucl.ac.uk
}

(Received 27 February 2006 and in revised form 2 September 2006)

Non-dispersive and weakly dispersive single-layer flows over axisymmetric obstacles, of non-dimensional height $M$ measured relative to the layer depth, are investigated. The case of transcritical flow, for which the Froude number $F$ of the oncoming flow is close to unity, and that of supercritical flow, for which $F>1$, are considered. For transcritical flow, a similarity theory is developed for small obstacle height and, for non-dispersive flow, the problem is shown to be isomorphic to that of the transonic flow of a compressible gas over a thin aerofoil. The non-dimensional drag exerted by the obstacle on the flow takes the form $D(\Gamma) M^{5 / 3}$, where $\Gamma=(F-1) M^{-2 / 3}$ is a transcritical similarity parameter and $D$ is a function which depends on the shape of the 'equivalent aerofoil' specific to the obstacle. The theory is verified numerically by comparing results from a shock-capturing shallow-water model with corresponding solutions of the transonic small-disturbance equation, and is found to be generally accurate for $M \lesssim 0.4$ and $|\Gamma| \lesssim 1$. In weakly dispersive flow the equivalent aerofoil becomes the boundary condition for the Kadomtsev-Petviashvili equation and (multiple) solitary waves replace hydraulic jumps in the resulting flow patterns.

For $\Gamma \gtrsim 1.5$ the transcritical similarity theory is found to be inaccurate and, for small $M$, flow patterns are well described by a supercritical theory, in which the flow is determined by the linear solution near the obstacle. In this regime the drag is shown to be $c_{d} M^{2} /\left(F \sqrt{F^{2}-1}\right)$, where $c_{d}$ is a constant dependent on the obstacle shape. Away from the obstacle, in non-dispersive flow the far-field behaviour is known to be described by the $N$-wave theory of Whitham and in dispersive flow by the Kortewegde Vries equation. In the latter case the number of emergent solitary waves in the wake is shown to be a function of $\mathscr{A}=3 M /\left(2 \delta^{2} \sqrt{F^{2}-1}\right)$, where $\delta$ is the ratio of the undisturbed layer depth to the radial scale of the obstacle.

\section{Introduction}

The flow of a single shallow layer of fluid over a three-dimensional (i.e. $h=h(x, y)$ ) obstacle has been investigated experimentally (e.g. by Lamb \& Britter 1984), largely because of its importance to the meteorological and oceanographic communities as a simple model of observed flows around islands, mountains, capes and sea-mounts (see e.g. Schär \& Smith 1993a). In the shallow-water limit (or 'non-dispersive' limit, as the phase speed of generated gravity waves is independent of wavenumber) the flow behaviour is typically characterized using two parameters, the non-dimensional 
obstacle height $M$ measured as the ratio of height to layer depth, and the Froude number $F$, the ratio of the undisturbed flow speed to the gravity-wave speed. In terms of these parameters, non-dispersive shallow-water models give qualitative, and sometimes reasonable quantitative, predictions for various observed phenomena, including vortex-shedding periods (Schär \& Smith 1993b) and wake lengths (Smith \& Smith 1995). The above studies, however, concentrated primarily on flows where the upstream velocity is subcritical $(F \lesssim 1)$. The behaviour of a supercritical oncoming flow $(F>1)$ has received less attention overall, although the different possible behaviour regimes were identified and investigated by Jiang \& Smith (2000). In particular they showed that, for sufficiently large $F$, linear theory accurately predicts the flow patterns near an axisymmetric obstacle even at relatively large values of $M$. This result was shown to hold even when the flow exhibited strong nonlinearity in the wakes to the sides of the obstacle. For $F$ closer to unity, however, the flow was found to become transcritical and the numerical solutions exhibited phenomena such as upstream 'bow shocks' and downstream 'V-waves'. The resulting flow patterns appeared very similar to those observed for the transonic flow of a compressible gas around an aerofoil, as might be expected from the well-known correspondence between the shallow-water equations and the equations of compressible-gas dynamics.

An important caveat in the interpretation of the Jiang \& Smith (2000) transcritical results, however, concerns the validity of the mass- and momentum-conserving hydraulic jumps that characterize the shallow-water solutions, for example the "bow shocks'. Whilst these hydraulic jumps may sometimes be a good physical model for the situation at breaking waves (Mei 1989; Baines 1995), they are an appropriate model only for those physical situations where a regularizing dissipation dominates over dispersive effects on scales typical of the jump width.

In many geophysical and laboratory situations it is dispersion that dominates. For example, there are numerous observations of multiple solitary waves, which are a distinctively dispersive phenomenon, in the flow upstream of obstacles, both in atmospheric flows ahead of islands (Li et al. 2004; Badgley, Miloy \& Childs 1969; Burk \& Haack 1999) and in laboratory experiments (Maxworthy, Dhieres \& Didelle 1984; Johnson et al. 2006). In order to describe such dispersive phenomena, the third important physical parameter in the problem, the ratio $\delta$ of the layer depth and the obstacle width, must be treated as non-zero. Various approximations to the full Euler equations are appropriate in the limit of small but non-zero $\delta$, including both the weakly forced case (i.e. $M$ small), for which the flow evolution is described by a forced Korteweg-de Vries (KdV hereafter) equation and its two-dimensional relatives (Grimshaw \& Smyth 1986; Kadomtsev \& Petviashvili 1970; Akylas 1994), and also the case of finite forcing (Green \& Naghdi 1976; Choi \& Camassa 1999). Solitarywave generation by moving pressure distributions has been widely studied for the case of weak forcing, but principally with regard to the closely related problem of ship-wave generation (e.g. Mei 1976; Katsis \& Akylas 1987; Lee \& Grimshaw 1990; Li \& Sclavounos 2002). However, exactly how the ship-wave results carry over to the problem of flow over three-dimensional obstacles has yet to be made explicit. In the case of two-dimensional obstacles, it has been shown in Grimshaw \& Smyth (1986) and subsequent studies that transcritical flows over small obstacles are often unsteady, with solitary waves periodically propagating upstream from the obstacle. Johnson \& Vilenski (2004) looked at the intermediate problem of flow over a quasithree-dimensional obstacle, i.e. an obstacle which is elongated asymptotically in the cross-stream direction. Numerical results were used to categorize the resulting flow patterns into a supercritical V-wave regime, a soliton regime, an unsteady flow regime 
and a subcritical modulated-wavetrain regime as the Froude number of the oncoming flow was reduced.

The current study will focus on flow over three-dimensional obstacles in the transcritical $(F \sim 1)$ and supercritical $(F>1)$ flow regimes. Both non-dispersive (shallow-water) and weakly dispersive flows will be considered, and for simplicity attention will be restricted to axisymmetric obstacles. The above studies leave several interesting questions unanswered. For example, is there a relationship between nondispersive shallow-water flow patterns over an obstacle and the corresponding weakly dispersive flows? Are the resulting flows always steady? What determines whether any such steady flows are purely subcritical, purely supercritical or transcritical (i.e. containing a transition between regions of supercritical and subcritical flow)? Also, how does the shape of the obstacle influence the resulting flow? Are properties other than bulk properties such as the obstacle height and volume important in determining the steady-flow response? In addition to the consideration of different flow-pattern regimes, the results obtained are presented in terms of the drag exerted on the flow by the obstacle. Drag is of particular interest to oceanographers, climate modellers and researchers involved in the development of numerical weather-prediction models, because of the need to parameterize the drag exerted by orography with spatial scales below the model grid scale. The results herein have a wider importance in this context than the restriction to single-layer flow might suggest because, as has been pointed out in the context of two-dimensional flow (Grimshaw \& Smyth 1986; Rottman \& Einaudi 1993), many results in the transcritical regime exactly correspond to those obtained for stratified flows when a single trapped vertical mode is near resonance. Throughout, results from compressible-gas dynamics (e.g. Chapman 2000) and ship dynamics (e.g. Mei 1976) are exploited as far as possible, since many aspects are shared between the related fields.

In $\S 2$ the mathematical formulation of the problem is introduced and asymptotic theories for small obstacle height $M$ are derived for both transcritical and supercritical regimes. Dispersive effects can be captured naturally in each analysis, at the same order as the leading-order nonlinearity, by making a suitable scaling choice for the aspect ratio $\delta$. In $\S 3$, the transcritical asymptotic theory is tested by comparing numerical results of the transonic small disturbance (TSD) equation resulting from the theory with numerical solutions of a shock-capturing shallow-water model. Weakly dispersive flow is compared directly with the corresponding non-dispersive flow in the transcritical limit. The different regimes of validity of the transcritical and supercritical theories are determined, and the properties of non-dispersive and weakly dispersive obstacle wakes in the supercritical regime are investigated. Finally, in $\S 4$, conclusions are drawn.

\section{Asymptotic theories for small obstacle height}

\subsection{Physical scenario and model equations}

The physical scenario to be considered is that of a single layer of inviscid fluid under gravity $g$, with undisturbed depth $H$ and constant density $\rho_{0}$, flowing with an initially uniform speed $U$ from right to left over an axisymmetric obstacle of finite volume with maximum height $h_{m}$ and radial scale $L$. The radial height profile of the obstacle is described by a non-dimensional function $h(r)$, where $r$ is a radial coordinate in the horizontal plane scaled with $L$, the distance from the centre of the obstacle. Without loss of generality, $h(0)$ is taken to be unity, and the volume $\mathscr{V}$ of the obstacle is set 
Obstacle

$$
h(r)
$$

$$
K(x)
$$

$$
G(\mathscr{X})
$$$$
c_{d}
$$

Witch of Agnesi

$$
\frac{a^{3}}{\left(a^{2}+r^{2}\right)^{3 / 2}} \quad \frac{a^{3}}{a^{2}+x^{2}}
$$$$
\frac{-2 a^{3}}{\left(\mathscr{X}^{2}+a^{2}\right)^{2}}
$$$$
\frac{\pi a}{2}
$$

$(a=1 / 2)$

$$
\exp \left\{-\frac{r^{2}}{a^{2}}\right\}
$$

$$
\frac{\sqrt{\pi} a}{2} \exp \left\{-\frac{x^{2}}{a^{2}}\right\}
$$

Gaussian

$$
(a=1 / \sqrt{2})
$$

cone

$$
1-\frac{r}{a}
$$$$
\frac{\sqrt{a^{2}-x^{2}}}{2}
$$$$
-\frac{\sqrt{\pi}}{a} \exp \left\{-\frac{\mathscr{X}^{2}}{a^{2}}\right\}
$$

$$
(a=\sqrt{3 / 2})
$$

$$
(r<a)
$$$$
\begin{array}{r}
-\frac{1}{a} \cosh ^{-1}\left\{\frac{a}{\mid \mathscr{X}}\right\} \\
(|\mathscr{X}|<a)
\end{array}
$$

$$
\begin{gathered}
-\frac{x^{2}}{2 a} \log \left\{\frac{\sqrt{a^{2}-x^{2}}+a}{|x|}\right\} \\
(|x|<a)
\end{gathered}
$$

hemi-ellipsoid

$$
\sqrt{1-\frac{r^{2}}{a^{2}}}
$$

$$
\frac{\pi}{4 a}\left(a^{2}-x^{2}\right)
$$

$$
-\frac{\pi}{2 a}
$$

$$
\frac{\pi^{2} a}{3}
$$

$$
(a=\sqrt{3} / 2)
$$$$
(|x|<a)
$$

$$
(|\mathscr{X}|<a)
$$

paraboloid

$$
1-r^{2}
$$$$
\frac{2}{3}\left(1-x^{2}\right)^{\frac{3}{2}}
$$$$
-2 \sqrt{1-\mathscr{X}^{2}}
$$

$$
(r<1)
$$

$$
(|x|<1)
$$

$$
(|\mathscr{X}|<1)
$$

TABLE 1. Obstacle shapes investigated and related functions (see the main text). The five obstacles have unit height and are normalized to have the same volume as the paraboloid $(V=\pi / 2)$, using the given value of the non-dimensional constant $a . c_{t}$ is Catalan's constant $(\approx 0.916)$.

according to

$$
\mathscr{V}=2 \pi \int_{0}^{\infty} r h(r) \mathrm{d} r=\frac{\pi}{2} .
$$

These restrictions on $h(r)$ ensure that comparisons of results for different obstacle shapes are made between obstacles with identical height and volume. Table 1 lists five obstacle shapes to be considered as examples, namely an obstacle with a 'Witch of Agnesi'-like profile (Jiang \& Smith 2000), a Gaussian, a cone, a hemi-ellipsoid and a paraboloid. The value of the non-dimensional constant $a$ in each case is chosen to satisfy (2.1).

The non-dimensional Euler equations for the flow scenario described above are

$$
\begin{aligned}
\frac{\mathrm{D} u}{\mathrm{D} t} & =-p_{x}, \\
\frac{\mathrm{D} v}{\mathrm{D} t} & =-p_{y}, \\
\delta^{2} \frac{\mathrm{D} w}{\mathrm{D} t} & =-p_{z}-1, \\
u_{x}+v_{y}+w_{z} & =0,
\end{aligned}
$$


where the advective derivative $D / D t=\partial_{t}+(u-F) \partial_{x}+v \partial_{y}+w \partial_{z}$. The equations (2.2) are subject to the boundary conditions

$$
\left.\begin{array}{rlrl}
\eta_{t}+(u-F) \eta_{x}+v \eta_{y} & =w & & \text { on } z=1+\eta(x, y, t), \\
M\left[(u-F) h_{x}+v h_{y}\right] & =w & & \text { on } z=M h(x, y), \\
p & =0 & & \text { on } z=1+\eta(x, y, t) .
\end{array}\right\}
$$

The physical coordinates $(x, y, z)$ have been scaled with $(L, L, H)$, the total velocity $\boldsymbol{u}=(-F+u, v, w)$ with $(c, c, c H / L)$, where $c \equiv \sqrt{g H}$ is the long-gravity-wave speed, time $t$ with $L / c$, pressure $p$ with $\rho_{0} c^{2}$ and the free-surface displacement $\eta$ with $H$. The three non-dimensional parameters discussed above now appear explicitly in (2.2), (2.3): the aspect ratio $\delta=H / L$, the Froude number $F=U / c$ and the non-dimensional obstacle height $M=h_{m} / H$. Results will be presented in terms of $\delta, F$ and $M$ in all that follows.

Particular attention will be paid to the non-dispersive limit $(\delta=0)$ of $(2.2),(2.3)$, which leads to the shallow-water equations

$$
\left.\begin{array}{rl}
u_{t}+(u-F) u_{x}+v u_{y} & =-\eta_{x} \\
v_{t}+(u-F) v_{x}+v v_{y} & =-\eta_{y} \\
+[(u-F) \sigma]_{x}+[v \sigma]_{y} & =0
\end{array}\right\}
$$

where $\sigma=1+\eta-M h$ is the layer thickness. In order to model the physical situation of breaking waves, the solutions of (2.4) are typically regularized by including the possibility of mass- and momentum-conserving hydraulic jumps. Such jumps satisfy

$$
\left.\begin{array}{r}
-V[\sigma]_{-}^{+}+[\sigma \boldsymbol{u} \cdot \boldsymbol{n}]_{-}^{+}=0, \\
-V[\sigma \boldsymbol{u} \cdot \boldsymbol{n}]_{-}^{+}+\left[\sigma(\boldsymbol{u} \cdot \boldsymbol{n})^{2}+\frac{1}{2} \sigma^{2}\right]_{-}^{+}=0,
\end{array}\right\}
$$

where $\boldsymbol{n}$ is a horizontal unit vector normal to the jump and $V$ is the jump velocity in the direction of $\boldsymbol{n}$. Square brackets denote the difference in quantities evaluated upstream and downstream of the jump. Hydraulic jumps satisfying (2.5) are known to violate the energy-conservation property of (2.2), (2.3). Therefore, there is an implicit assumption that the non-dispersive limit of (2.2), (2.3) corresponds to a physical situation in which an unspecified dissipation acts on the (unresolved) horizontal scale of the jumps. Hence, as discussed above, there is an important distinction between the nondispersive $(\delta=0)$ solutions of $(2.2),(2.3)$ and solutions in the weakly dispersive $(\delta \rightarrow 0)$ limit, in which energy is conserved. Next, two different asymptotic regimes for small obstacle height $M$ will be considered: transcritical flow for which the Froude number $F$ is close to unity and supercritical flow for which $F>1$. In each case, dispersive effects are included by scaling $\delta^{2}$ with the free-surface displacement, which ensures that dispersion enters the analysis at the same order as nonlinearity in each problem.

\subsection{Transcritical flow: the equivalent-aerofoil regime}

The relevant scaling regime for transcritical flow over an obstacle with small height $M \ll 1$ is suggested by a derivation of the weakly two-dimensional $\mathrm{KdV}$ equation (Kadomtsev \& Petviashvili 1970, the KP equation hereafter) and the scaling regime adopted for transonic flow over a thin aerofoil (see e.g. Chapman 2000). On the basis of these previous works, the non-dimensional parameters

$$
\Gamma=(F-1) M^{-2 / 3}, \quad \Delta=\delta M^{-1 / 3}
$$

are taken to be of order unity. The parameter $\Gamma$ will be referred to as the transcritical similarity parameter (following the terminology of gas dynamics, see e.g. Chapman 
$2000)$ and is the relevant measure of the degree of super criticality $(F>1, \Gamma>0)$ or sub-criticality $(F<1, \Gamma<0)$ of the flow for a given obstacle height $M$. The parameter $\Delta$ is a measure of the relative importance of dispersion in the system; the nondispersive limit discussed above corresponds to $\Delta=0$ and the weakly dispersive limit corresponds to $\Delta \rightarrow 0$.

Introducing a small parameter $\epsilon=M^{2 / 3}$ and seeking solutions of (2.2), (2.3) that evolve on a 'slow' time scale $\tau=\epsilon t$, the variables $u, v, w, p$ and $\eta$ may be expanded in powers of $\epsilon^{1 / 2}$ as follows:

$$
\left.\begin{array}{rl}
u & =\epsilon\left(u_{0}+\epsilon^{1 / 2} u_{1}+\epsilon u_{2}+\cdots\right), \\
v & =\epsilon^{3 / 2}\left(v_{0}+\epsilon^{1 / 2} v_{1}+\epsilon v_{2}+\cdots\right), \\
w & =\epsilon\left(w_{0}+\epsilon^{1 / 2} w_{1}+\epsilon w_{2}+\cdots\right), \\
p & =1-z+\epsilon\left(p_{0}+\epsilon^{1 / 2} p_{1}+\epsilon p_{2}+\cdots\right), \\
\eta & =\epsilon\left(\eta_{0}+\epsilon^{1 / 2} \eta_{1}+\epsilon \eta_{2}+\cdots\right) .
\end{array}\right\}
$$

The expansions (2.7) are used to match leading-order asymptotic solutions of (2.2), (2.3) in two separate regions defined by their cross-stream distance from the centre of the obstacle at $y=0$. Note that the leading-order scaling for $v$ is $O\left(\epsilon^{3 / 2}\right) \equiv O(M)$, which is consistent with the weak two-dimensionality of the KP equation (Grimshaw $\&$ Melville 1989) and implies that the cross-stream velocity $v$ will enter at the same order as the forcing terms due to the obstacle.

In the inner region, which describes the flow on streamlines that pass over or near to the obstacle, the cross-stream coordinate is taken to be $y$. Denoting inner-region variables with the superscript $i$ and inserting the expansion (2.7) into (2.2), (2.3), at leading order it is established that

$$
u_{0}^{i}=p_{0}^{i}=\eta_{0}^{i}(x), \quad w_{0}^{i}=-\eta_{0 x}^{i} z .
$$

The leading-order solution is therefore described by an (as yet) undetermined $y$-independent function $\eta_{0}^{i}(x)$, except for the leading-order cross-stream velocity $v_{0}^{i}$. At the next order in the expansion we have

$$
u_{1}^{i}=p_{1}^{i}=\eta_{1}^{i}(x, y), \quad w_{1}^{i}=-\left(\eta_{1 x}^{i}+v_{0 y}^{i}\right) z-h_{x} .
$$

Inserting the above expression for $w_{1}^{i}$ into the kinematic condition at the free surface gives $v_{0 y}^{i}=-h_{x}$. Integrating in $y$ and using the fact that $v=0$ on $y=0$ gives

$$
v_{0}^{i}(x, y)=-\int_{0}^{y} h_{x}(x, \hat{y}) \mathrm{d} \hat{y},
$$

the velocity $v_{0}^{i}$ being related to the free-surface height through

$$
v_{0 x}^{i}=\eta_{1 y}^{i} .
$$

Proceeding to higher order, it becomes clear that $\eta_{0}^{i}(x)$ is not now determined by the inner-region expansion alone. Furthermore, the solution becomes invalid away from the obstacle where $|y| \sim O\left(\epsilon^{-1 / 2}\right)$. At these distances, higher-order terms in the series expansion become comparable with those at leading order. This indicates that $\eta_{0}^{i}$ must be determined by matching with an outer-region solution which is valid for $|y| \sim O\left(\epsilon^{-1 / 2}\right)$.

In the outer region the variable $Y=\epsilon^{1 / 2} y$ is introduced to capture the cross-stream scale on which the leading-order free-surface height and streamwise velocity vary in the direction perpendicular to the oncoming flow. The influence of the obstacle is 
experienced only through the boundary condition at $Y=0$. Throughout the rest of the domain in the rescaled coordinates, the bottom boundary is taken to be flat. In the outer region we seek far-field asymptotic solutions, denoted by a superscript $o$, of (2.2), (2.3) by substituting

$$
u_{0}=u_{0}^{o}(x, Y, z, \tau) \quad \text { etc. }
$$

for each variable in the series expansion (2.7). At leading order,

$$
u_{0}^{o}=p_{0}^{o}=\eta_{0}^{o}(x, Y), \quad w_{0}^{o}=-\eta_{0 x}^{o} z,
$$

together with

$$
v_{0 x}^{o}=\eta_{0 Y}^{o} .
$$

At the next order, $u_{1}^{o}, v_{1}^{o}, w_{1}^{o}, p_{1}^{o}, \eta_{1}^{o}$ are found to satisfy a set of equations identical to (2.12), (2.13) and hence may be set to zero without loss of generality. To obtain an equation for $\eta_{0}^{o}$ we must proceed to $O\left(\epsilon^{2}\right)$, thereby introducing nonlinear terms. The derivation from here follows the standard derivation of the KP equation (see e.g. Grimshaw \& Melville 1989). The following relations between the $O\left(\epsilon^{2}\right)$ terms are obtained:

$$
\left.\begin{array}{l}
p_{2}^{o}=\eta_{2}^{o}+\frac{\Delta^{2}}{2} \eta_{0 x x}^{o}\left(1-z^{2}\right), \\
u_{2 x}^{o}=\eta_{2 x}^{o}+\frac{\Delta^{2}}{2} \eta_{0 x x x}^{o}\left(1-z^{2}\right)+\eta_{0 \tau}^{o}-\Gamma \eta_{0 x}^{o}+\eta_{0}^{o} \eta_{0 x}^{o}, \\
w_{2}^{o}=z\left(-\eta_{2 x}^{o}-\frac{\Delta^{2}}{2} \eta_{0 x x x}^{o}-v_{0 Y}^{o}-\eta_{0 \tau}^{o}+\Gamma \eta_{0 x}^{o}-\eta_{0}^{o} \eta_{0 x}^{o}\right)+z^{3} \frac{\Delta^{2}}{6} \eta_{0 x x x}^{o} .
\end{array}\right\}
$$

Inserting the above expression for $w_{2}^{o}$ into the kinematic condition at the free surface reveals that terms involving $\eta_{2}$ cancel, to give

$$
\left(2 \eta_{0 \tau}^{o}+3 \eta_{0}^{o} \eta_{0 x}^{o}-2 \Gamma \eta_{0 x}^{o}+\frac{\Delta^{2}}{3} \eta_{0 x x x}^{o}\right)_{x}+\eta_{0 Y Y}^{o}=0,
$$

where $v_{0}^{o}$ has been eliminated using (2.13). Equation (2.15) is the KP equation, as might be anticipated for the outer region. The novel component is the derivation of the appropriate boundary condition on $Y=0$, which is obtained by matching with the inner solution. This results in the following conditions:

$$
\left.\begin{array}{rl}
\eta_{0}^{i}(x) & =\lim _{Y \rightarrow 0} \eta_{0}^{o}(x, Y), \\
\lim _{y \rightarrow \pm \infty} \eta_{1 y}^{i}(x, y) & =\lim _{Y \rightarrow 0^{ \pm}} \eta_{0 Y}^{o}(x, Y), \\
\lim _{y \rightarrow \pm \infty} v_{0}^{i}(x, y) & =\lim _{Y \rightarrow 0^{ \pm}} v_{0}^{o}(x, Y) .
\end{array}\right\}
$$

Using (2.10) and (2.11) the matching conditions may be replaced by the single condition

$$
\left[\eta_{0 Y}^{o}\right]_{-}^{+}=-\int_{-\infty}^{\infty} h_{x x}(x, \hat{y}) \mathrm{d} \hat{y},
$$

where the square brackets denote the difference between evaluation at $Y \rightarrow 0^{+}$and $Y \rightarrow 0^{-}$. Note that for obstacles symmetric about $y=0$, including axisymmetric obstacles, the boundary condition (2.17) for equation (2.15) can be replaced by a 'line-charge' forcing term on the right-hand side of (2.15) of the form

$$
F(x, Y)=-2 \delta(Y) K_{x x}(x), \quad \text { where } K(x)=\int_{0}^{\infty} h(x, \hat{y}) \mathrm{d} \hat{y}
$$

and $\delta(Y)$ is the Dirac delta function. 

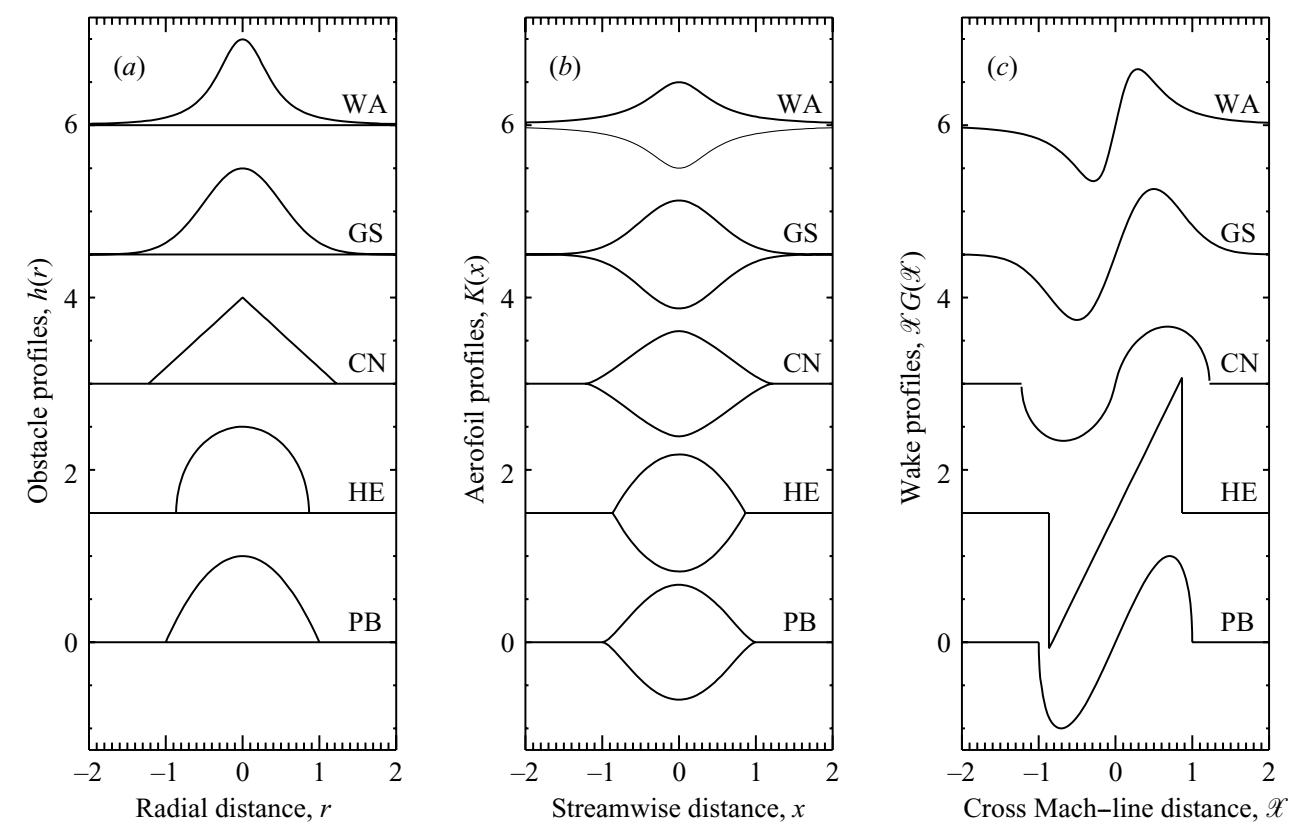

FIgURE 1. (a) Obstacle profiles $h(r)$ for the 'Witch of Agnesi' obstacle (WA), the Gaussian (GS), the cone $(\mathrm{CN})$, the hemi-ellipsoid (HE) and the paraboloid (PB). (b) The equivalent aerofoils $K(x)$ for the obstacles. $(c)$ The supercritical, linear, far-field wake-height fields $-\mathscr{X} G(\mathscr{X})$ for the obstacles. See table 1 for details.

To solve the non-dispersive $(\Delta=0) \mathrm{KP}$ equation, it is convenient to introduce

$$
\phi(x, Y)=\int_{x}^{\infty} \eta_{0}^{o}(\hat{x}, Y) \mathrm{d} \hat{x},
$$

allowing (2.15) to be integrated to give the following system, valid in the half-plane $Y \geqslant 0$,

$$
\left(-2 \Gamma-3 \phi_{x}\right) \phi_{x x}+\phi_{Y Y}=0, \quad \text { with } \phi_{Y}(x, 0)=K_{x}(x) \text { on } Y=0 .
$$

This equation may be recognised as the transonic small disturbance (TSD hereafter) equation, or Kármán-Guderley equation, for flow over a thin symmetric aerofoil with half-thickness $\epsilon K(x)$. The 'equivalent aerofoil' half-thickness functions $K(x)$ for the five obstacles chosen as examples are given in table 1 and the aerofoil profiles themselves are plotted in figure 1 .

The transcritical asymptotic theory presented above has the advantageous effect of reducing the three-parameter $(M, F, \delta)$ problem for flow over an obstacle of arbitrary height to a two-parameter problem $(\Gamma, \Delta)$ valid for small obstacles $M \ll 1$. In particular, in the case of non-dispersive flow $(\delta=\Delta=0)$ the transcritical theory yields a one-parameter problem represented by the TSD equation (2.19), which has been widely studied in the gas dynamics literature. Highly developed numerical methods exist to determine accurate steady solutions to (2.19) and analytic similarity solutions also exist, (e.g. Chapman 2000), although these do not seem to be easily related to the flow patterns forced by a particular obstacle. By examining the equivalent-aerofoil thickness profile $K(x)$ of a given obstacle, insight from engineering studies of flow over aerofoils may be used to anticipate the resulting flow patterns as well as the 
drag exerted by the obstacle on the flow. The drag on the obstacle, given by

$$
\mathscr{D}=M \int_{-\infty}^{\infty} \int_{-\infty}^{\infty} p(x, y, h) h_{x}(x, y) \mathrm{d} x \mathrm{~d} y,
$$

with dimensional units $\rho_{0} g H^{2} L$, can be shown at leading order to be equal to

$$
\mathscr{D}=M^{5 / 3} \int_{-\infty}^{\infty} \eta_{0}^{o}(x, 0) K_{x}(x) \mathrm{d} x=M^{5 / 3} D(\Gamma),
$$

i.e. $M^{5 / 3}$ times the non-dimensional drag on the equivalent aerofoil in the TSD solution, which is denoted $D(\Gamma)$.

In $\S 3$, the range of validity of the transcritical theory is investigated numerically for the case of non-dispersive flow. In particular, one further aspect of non-dispersive flow over three-dimensional obstacles that has received considerable previous attention (Schär \& Smith 1993a; Baines 1995, figure 2.30 therein) is the location of the boundaries between transcritical and supercritical flow, and between transcritical and subcritical flow, as plotted on an $(M, F)$-diagram. A transcritical flow in this sense simply describes a supercritical flow containing an embedded region of subcritical flow, or a subcritical flow with an embedded region of supercritical flow. The boundaries in $(M, F)$ parameter space described above are boundaries at which the embedded region appears or vanishes. In the transcritical limit, the boundaries must exist at fixed values, $\Gamma=\Gamma_{-}<0$ and $\Gamma=\Gamma_{+}>0$, since the TSD equation (2.19) depends only on the single parameter $\Gamma$. Hence for small obstacle height $M$, the transcritical theory predicts that the regime boundaries occur at

$$
F=1+\Gamma_{-} M^{2 / 3} \quad \text { and } \quad F=1+\Gamma_{+} M^{2 / 3} \quad\left(\Gamma_{-}<0<\Gamma_{+}\right),
$$

where $\Gamma_{-}$and $\Gamma_{+}$are constants that must be determined from numerical solutions of (2.19) for each obstacle's equivalent aerofoil $K(x)$. The accuracy of the predictions (2.21) for the regime boundaries is examined in $\S 3$.

\subsection{Supercritical flow}

Here, an asymptotic theory for the flow past an obstacle is presented that is formally valid for $M \ll 1, F-1 \gg M^{2 / 3}$ (or $\Gamma \gg 1$ in the terms of the previous section). As with transcritical flow, the asymptotic theory is obtained by matching between an inner region near the obstacle and an outer far field. Aspects of the theory have been developed and discussed elsewhere (Gurevich et al. 1995, 1996; Jiang \& Smith 2000; Johnson \& Vilenski 2004), so the aim of the current section is to apply these previous results to the specific problem under discussion, place it in context with the transcritical theory above and introduce some new results specific to the case of axisymmetric obstacles.

For non-dispersive and weakly dispersive flow with $\delta \sim M^{1 / 2}$, the inner-region solution of (2.2), (2.3) in the vicinity of the obstacle is given by linear theory and has been discussed by Baines (1995) and Jiang \& Smith (2000). Introducing $\varepsilon=M$ as a small parameter and expanding,

$$
\left.\begin{array}{rl}
u & =\varepsilon\left(u_{0}+\varepsilon u_{2}+\cdots\right) \\
v & =\varepsilon\left(v_{0}+\varepsilon v_{2}+\cdots\right) \\
w & =\varepsilon\left(w_{0}+\varepsilon w_{2}+\cdots\right) \\
p & =1-z+\varepsilon\left(p_{0}+\varepsilon p_{2}+\cdots\right) \\
\eta & =\varepsilon\left(\eta_{0}+\varepsilon \eta_{2}+\cdots\right)
\end{array}\right\}
$$


the leading-order solution is found to satisfy

$$
\left(F^{2}-1\right) \eta_{0 x x}^{l}-\eta_{0 y y}^{l}=F^{2} h_{x x},
$$

where the superscript $l$ has been introduced to denote the inner-region solution for the supercritical problem. Using Green's function techniques, Jiang and Smith obtained the general solution (adapted here for right-to-left flow)

$$
\eta_{0}^{l}(x, y)=\frac{F^{2}}{2 \gamma}\left\{\int_{-\infty}^{y} h_{x}(-x-\gamma(y-\tilde{y}), \tilde{y}) \mathrm{d} \tilde{y}+\int_{y}^{\infty} h_{x}(-x+\gamma(y-\tilde{y}), \tilde{y}) \mathrm{d} \tilde{y}\right\},
$$

where $\gamma=\sqrt{F^{2}-1}$. It is useful at this stage to introduce a rotated coordinate system $\left(\mathscr{X}_{ \pm}, \mathscr{Y}_{ \pm}\right)=(x \pm \gamma y, y \mp \gamma x) / F$ for the regions $y>0$ and $y<0$ respectively. In $y>0$, the new coordinate system is rotated anticlockwise through an angle $\tan ^{-1} \gamma$, so that $\mathscr{X}_{+}$ measures distance perpendicular to the Mach lines given by $x+\gamma y=$ constant, and $\mathscr{Y}_{+}$measures the distance along these Mach lines. A similar, but clockwise, rotation occurs for the coordinates in $y<0$. In terms of these new coordinates, Johnson \& Vilenski (2004) noted that provided the obstacle height decays sufficiently rapidly at large distances then as $y \rightarrow \pm \infty$ the solution (2.24) converges to

$$
\lim _{y \rightarrow \pm \infty} \eta_{0}^{l}(x, y)=\frac{F^{2}}{2 \gamma^{2}} \int_{-\infty}^{\infty}\left\{\begin{array}{cl}
h_{x}\left(\xi,\left(F \mathscr{X}_{+}+\xi\right) / \gamma\right) \mathrm{d} \xi & y \rightarrow+\infty \\
h_{x}\left(-\xi,\left(\xi-F \mathscr{X}_{-}\right) / \gamma\right) \mathrm{d} \xi & y \rightarrow-\infty
\end{array}\right.
$$

Equation (2.25) shows that the linear solution away from the obstacle tends to a function of the single coordinate $\mathscr{X}_{ \pm}$, the sign being taken according to whether we are looking at $y \rightarrow \pm \infty$. For definiteness, the upper half-plane $y>0$ will be considered hereafter (the lower half-plane solution being symmetric), and the subscript + will be dropped.

For the particular case of an axisymmetric obstacle $h=h(r)$, a change of variables in the expression (2.25) results in

$$
\eta_{0}^{l}(\mathscr{X}) \rightarrow-\frac{1}{\gamma} \mathscr{X} G(\mathscr{X}) \quad \text { where } G(\mathscr{X})=\int_{|\mathscr{X}|}^{\infty} \frac{h^{\prime}(r)}{\sqrt{r^{2}-\mathscr{X}^{2}}} \mathrm{~d} r .
$$

The drag $\mathscr{D}$ on the obstacle is easily shown to be equal to the sum of the momentum fluxes of the outgoing waves. Hence, from symmetry considerations and on the basis of the solution at large $y>0$,

$$
\mathscr{D}=2 M^{2} \int_{-\infty}^{+\infty} u_{0}^{l} v_{0}^{l} \mathrm{~d} x=2 M^{2} F \int_{-\infty}^{+\infty} u_{0}^{l}(\mathscr{X}) v_{0}^{l}(\mathscr{X}) \mathrm{d} \mathscr{X} .
$$

It is clear from the linearized momentum equations that the velocities $u_{0}^{l}(\mathscr{X})$ and $v_{0}^{l}(\mathscr{X})$ satisfy $F u_{0}^{l}=\eta_{0}^{l}$ and $F v_{0}^{l}=\gamma \eta_{0}^{l}$ in the far field. Substituting into (2.27) gives

$$
\mathscr{D}=c_{d} \frac{M^{2}}{F \sqrt{F^{2}-1}},
$$

where the constant $c_{d}$ is given by

$$
c_{d}=2 \int_{-\infty}^{\infty} \mathscr{X}^{2} G(\mathscr{X})^{2} \mathrm{~d} \mathscr{X} .
$$

Jiang \& Smith (2000) gave the result (2.28) for the specific example of the 'Witch of Agnesi' obstacle described in table 1. Here it has been shown that (2.28) is the general form for the drag for all axisymmetric obstacles. The integrals in (2.26) and (2.29) are 
typically straightforward, and the results for the five example obstacles are given in table 1 . The general shape of the linear far-field wave, given by $-\mathscr{X} G(\mathscr{X})$, is plotted in figure 1 for each obstacle. It is notable from table 1 that the relatively compact obstacles (e.g. the hemi-ellipsoid or paraboloid) have significantly higher linear drags (higher $c_{d}$ ) compared with those for distributed obstacles (e.g. the Witch of Agnesi).

Supercritical flows are linear only in the vicinity of the obstacle because, in the absence of explicit dissipation, the shallow-water waves generated at the obstacle will steepen and break as they propagate (see e.g. Whitham 1974), regardless of their initial amplitude. This behaviour can be examined by seeking far-field steady solutions of (2.2), (2.3). In order to determine the behaviour of the solution further from the obstacle, 'stretched' variables $\left(\mathscr{X}_{*}, \mathscr{Y}_{*}\right)=\varepsilon(\mathscr{X}, \mathscr{Y})$ can be introduced. Expanding (2.2), (2.3) in powers of $\varepsilon$, according to (2.22), derivatives in the $\mathscr{X}$ - and $\mathscr{Y}$-directions are found to appear at different orders, and it is thereby natural to seek solutions of the form $\eta=\eta^{f}\left(\mathscr{X}, \mathscr{Y}_{*}\right)$ etc. The superscript $f$ denotes the supercritical far-field solutions. To ensure that dispersion enters the problem at the correct order, it is taken that the variable

$$
\bar{\Delta}=\delta M^{-1 / 2},
$$

is of order unity. At leading order, (2.2), (2.3) are satisfied by

$$
u_{0}^{f}=\frac{\eta_{0}^{f}}{F}, \quad v_{0}^{f}=\frac{\gamma \eta_{0}^{f}}{F}, \quad p_{0}^{f}=\eta_{0}^{f}, \quad w_{0}^{f}=-\eta_{0 x}^{f} z .
$$

To find an equation for $\eta_{0}^{f}$, it is necessary to proceed to the next order, where it can be shown from (2.2) that

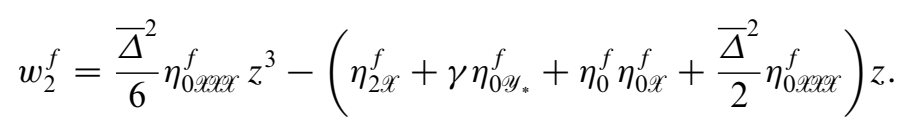

Using this expression for $w_{2}^{f}$ in the kinematic condition at the free surface in (2.3), terms involving $\eta_{2}^{f}$ are found to cancel and a far-field equation for the leading-order height field $\eta_{0}^{f}$ is obtained:

$$
2 \gamma \eta_{0 \mathscr{y}_{*}}^{f}+3 \eta_{0}^{f} \eta_{0 \mathscr{X}}^{f}+\frac{\bar{\Delta}^{2}}{3} \eta_{0 \mathscr{x} x}^{f}=0 .
$$

Equation (2.31) is the familiar KdV equation, in which the 'stretched' space variable $\mathscr{Y}_{*}$ assumes the role of the time-like variable.

The 'initial' condition for (2.31) is determined by the matching condition with the inner solution (2.24),

$$
\lim _{\mathscr{Y}_{*} \rightarrow 0^{+}} \eta_{0}^{f}=\lim _{y \rightarrow+\infty} \eta_{0}^{l}
$$

or, from (2.25),

$$
\eta_{0}^{f}(\mathscr{X}, 0)=-\frac{1}{\gamma} \mathscr{X} G(\mathscr{X})
$$

with $G(\mathscr{X})$ defined by (2.26). Johnson \& Vilenski (2004) previously derived equation (2.31) for the case of supercritical flow over an elongated ridge in the weakly dispersive limit (the Kadomtsev-Petviashvili limit, see (2.15)). Here, it has been shown that the solution applies equally to the more general problem of supercritical flow over a small isolated obstacle $(M \ll 1)$ at all Froude numbers satisfying $F>1, F-1 \gg M^{2 / 3}$. 


\section{Validity of the asymptotic theories and behaviour at finite obstacle height}

\subsection{Numerical models used to test the asymptotic theory}

Three separate numerical models have been used to investigate the range of validity of the asymptotic theories by comparing the nonlinear behaviour at finite $M$ to that predicted by the transcritical asymptotic theory. The numerical models were designed to solve the shallow-water equations (2.4), the TSD equation (2.19) and the KP equation (2.15), respectively. In practice, throughout most of the domain all three equations are hyperbolic when the oncoming flow is supercritical $(F>1, \Gamma>0)$ and elliptic when the oncoming flow is subcritical. As critical flow is approached $(F \rightarrow 1$, $\Gamma \rightarrow 0$ ), a progressively larger computational domain and progressively longer timescale of integration are required to obtain solutions that are steady in the vicinity of the obstacle. There are two main reasons that prevent steady solutions, where they exist, being found for near-critical oncoming flow, as follows.

(a) Propagation of the solution at sufficient amplitude to the upstream boundary may occur, thereby either invalidating the inflow condition or eventually interfering with the solution near the obstacle in the periodic domain. Overcoming this problem necessitates increasing the size of the domain in the $x$-direction as $F$ approaches unity $(\Gamma \rightarrow 0)$.

(b) Propagation of the solution at sufficient amplitude to the lateral boundaries may occur, causing reflected waves or hydraulic jumps to interfere with the solution in the vicinity of the obstacle. To prevent this, the size of the domain in the $y$-direction must also be increased as $F$ approaches unity $(\Gamma \rightarrow 0)$.

For these reasons a range of domain sizes was used with each model. For all the numerical calculations, care was taken on a case-by-case basis to ensure that the flow patterns and calculated drags were unaffected by further increases in domain size, that the flow field in the vicinity of the obstacle was sufficiently steady and that adequate convergence with respect to spatial resolution occurred.

For the shallow-water equations, the model chosen was the CLAWPACK finitevolume code (Conservation LAWs software PACKage, LeVeque 2002), which is designed to solve hyperbolic systems of equations, usually written in conservation form. CLAWPACK uses an algorithm due to Roe (1981) in which the global problem under investigation is discretized into a set of local Riemann problems by assuming a piecewise-discontinuous form for the solution. The set of Riemann problems is then linearized and solved using Godunov's upwind method (LeVeque 2002). The great advantage of this model is that, provided the governing equations can be written in conservation form, the speed of propagation of shock discontinuities that satisfy the global conservation laws can be captured accurately. For the current work CLAWPACK was adapted to solve the shallow-water equations (2.4) written in conservation form, and the relevant shock discontinuities were taken to be the hydraulic jumps (2.5). Note that the forcing terms due to the obstacle cannot be included in the conservation form of (2.4) and are handled using the method of Strang splitting, for which the time tendency at each step is split into a contribution from the unforced equations, calculated using Roe's method, and a separately computed contribution from the forcing, evaluated using standard finite-difference methods. Symmetry considerations permit the solution to be calculated on the half-plane $y \geqslant 0$, and solutions are obtained on domains ranging in size from $10 L \times 10 L$ to $60 L \times 45 L$, with grid-spacing ranging between $\delta x=0.1 L$ (low resolution) to $\delta x=0.01 L$ (high resolution). The assumption of symmetry about $y=0$ would be inappropriate if there were a possibility that the obstacle wake might be unstable (Schär \& Smith 1993b); 
however, this is not the case for the results presented here. Outflow conditions are imposed at the remaining boundaries, following Schär \& Smith (1993a). The model is integrated forwards in time with an adjustable time step based on the Courant-Friedrichs-Lewy criterion (for details see, LeVeque 2002) until the flow in a predetermined region around the obstacle converges to satisfy a steady-state numerical criterion.

The second numerical model was used to find steady solutions of the TSD equation (2.19) subject to forcing from the obstacle's equivalent aerofoil (2.18). In order to perform an entirely independent test on the CLAWPACK shallow-water results, a different set of numerical techniques from the gas-dynamics literature was used. The numerical algorithm employed was a type-dependent finite-difference scheme, as developed by Murman \& Cole (1971) and refined by Engquist \& Osher (1980). The basic concept behind the technique is that a different finite-difference stencil is used depending on whether the flow is locally subsonic so that (2.19) is locally elliptic (centred differences), locally supersonic so that (2.19) is hyperbolic (upstream-sided differences) or in transition between the two at a shock or sonic line. The resulting set of difference equations is solved using the monotonic implicit approximate-factorization scheme of Goorjian \& Van Buskirk (1981), with the lowfrequency modes damped by means of the multigrid acceleration technique described by Jameson (1979). For a supercritical oncoming flow, the boundary conditions are $\phi_{x}=0$ at the upstream boundary, with outflow conditions on the upper and downstream boundaries. For a subsonic oncoming flow, $\phi_{x}$ and $\phi_{y}$ are specified on the boundaries using the anticipated asymptotic form for the far-field solution of the TSD (e.g. Cole \& Cook 1986). The high level of sophistication of the algorithm allows steady solutions of the TSD to be found on a high-resolution grid $(2049 \times 2049$ to $4097 \times 4097$ grid points) within a few minutes on a workstation. The domain sizes used are $40 L \times 40 \epsilon^{-1 / 2} L$ to $60 L \times 60 \epsilon^{-1 / 2} L$, increasing as $\Gamma$ approaches zero.

The third numerical model, used to solve the KP equation (2.15), is a standard pseudo-spectral model similar to those described by Fornberg \& Driscoll (1999) and Johnson \& Vilenski (2004). Equation (2.15) is solved on a doubly periodic domain with size either $200 L \times 50 \epsilon^{-1 / 2} L$ (small domain) or $400 L \times 50 \epsilon^{-1 / 2} L$ (large domain). The numerical resolutions used for the solutions shown are $2048 \times 512$ (small domain) and $4096 \times 512$ (large domain) Fourier modes, corresponding to a grid spacing of approximately $0.02 L \times 0.02 \epsilon^{-1 / 2} L$. In order to avoid numerical problems due to the excitation of Fourier modes associated with the grid scale, the Dirac-delta-function forcing implied by (2.18) is replaced by a Gaussian function $(\hat{\alpha} / \sqrt{\pi} \delta Y) \exp \left\{-\hat{\alpha}^{2} Y^{2} / \delta Y^{2}\right\}$, which is equivalent to the Dirac delta function as the grid spacing $\delta Y \rightarrow 0$. At sufficiently high resolution, the results were found to be independent of the constant $\hat{\alpha}$ over a range of values, with $\hat{\alpha}=1$ used in the calculations shown. The model is integrated forwards in time using a predictorcorrector method with time step $\Delta \tau=0.02$ until either a steady state criterion is attained for the flow in the vicinity of the obstacle or waves generated by the obstacle are found to have propagated throughout the domain, causing interference with the solution at the obstacle and preventing further convergence. Integrations with $\Gamma$ close to zero were invariably affected by the latter problem.

\subsection{Non-dispersive transcritical flow}

In the limit of small $M$, non-dispersive transcritical flow is described by the TSD equation (2.19) with the equivalent aerofoil boundary condition (2.18). In this limit, non-dispersive shallow-water flow over an obstacle, which is a problem having two 

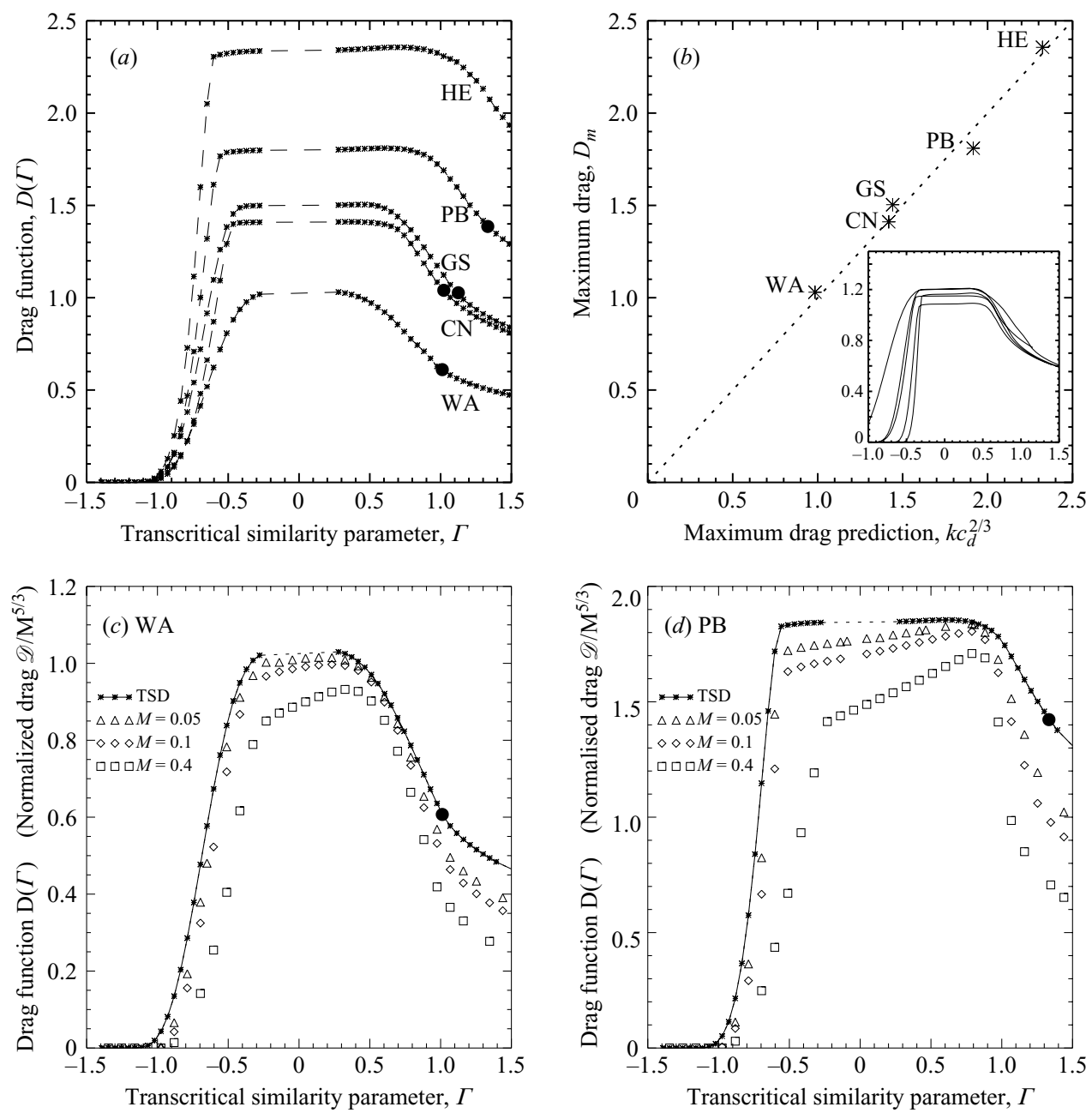

FIGURE 2. The drag function $D(\Gamma)$ vs. the transcritical similarity parameter $\Gamma$, as calculated from a series of steady numerical solutions of the TSD (2.19). (a) Results for the five obstacles detailed in table 1: 'Witch of Agnesi' (WA), Gaussian (GS), cone (CN), paraboloid (PB) and hemi-ellipsoid (HE). The solid dot on each curve shows the location of $\Gamma_{+}$, and for the dashed region of each curve there is no numerical data because of the difficulty in obtaining results for $F$ close to unity. $(b)$ The correlation between the rescaled supercritical drag coefficient $k c_{d}^{2 / 3}$ and the maximum transcritical drag $\mathscr{D}_{m}$ for the five obstacles. The inset, in which $\mathscr{D} / c_{d}^{2 / 3}$ is plotted vs. $\Gamma c_{d}^{-2 / 3}$, shows the extent to which the five drag curves collapse onto a single profile after application of the scaling described in the text. $(c)$ The 'Witch of Agnesi' (WA) and $(d)$ paraboloid (PB) results. The corresponding normalized drags $\mathscr{D} / M^{5 / 3}$ from the shallow-water-model numerical calculations are plotted on $(c, d)$ for comparison; the results for obstacle heights $M=0.05,0.1$ and 0.4 are shown.

parameters $(M, F)$, is reduced to the single-parameter $(\Gamma)$ aerofoil problem. In this section, the accuracy of predictions for drag and flow patterns from the aerofoil problem are tested by comparison with numerical calculations of the full shallowwater equations with an obstacle of finite height $M$, as described above.

Figure 2 shows the drag function $D(\Gamma)$ calculated from a series of steady numerical solutions of the TSD equation (2.19) with values of $\Gamma$ in the range $[-1.5,1.5]$. 
Figure 2(a) shows the drag function $D(\Gamma)$ calculated for each of the five equivalent aerofoils $K(x)$ corresponding to the five obstacles detailed in table 1. As might perhaps have been anticipated from the engineering literature, the shape of the drag curve is found to be remarkably similar for the different aerofoils. As $\Gamma$ increases, the drag becomes non-zero when a hydraulic jump and supercritical region first appear at $\Gamma=\Gamma_{-} \approx-1.2$. As $\Gamma$ increases further, the drag increases until it reaches a plateau region of values very close to the maximum attained. For $\Gamma>0.8$, the drag begins to decrease, and the flow eventually becomes entirely supercritical at $\Gamma=\Gamma_{+}$, which lies in the range 1.0-1.75 and is marked as a solid dot on each curve. At $\Gamma=\Gamma_{+}$the drag is typically around $60 \%-70 \%$ of its peak value. Both the peak drag $D_{m}$ and the value of $\Gamma_{+}$, which marks the boundary between supercritical and transcritical flow, vary significantly between the different obstacles. Calculated values of $\Gamma_{-}, \Gamma_{+}$and $D_{m}$ are given in table 2 , and it is clear that for transcritical flow, just as for supercritical flow, compact obstacles such as the hemi-ellipsoid and paraboloid exert more drag on the flow than distributed obstacles such as the 'Witch of Agnesi'.

Can the peak transcritical drag $D_{m}$ be estimated from some easily calculable property of the obstacle shape function $h(r)$ ? The strong degree of similarity between the shapes of the five curves in figure $2(a)$ suggests that some simple rescaling arguments may be useful. Changes in the transcritical drag can be seen to be closely related to changes in the supercritical drag coefficient $c_{d}$, defined in (2.29). As $c_{d}$ increases (see table 1), $D_{m}$ and the range of $\Gamma$ for which $D(\Gamma)$ is uniformly large both increase. One possibility is that the drag curves $D(\Gamma)$ collapse to some 'universal' drag curve under the rescalings $\Gamma \rightarrow \tilde{\Gamma} c_{d}^{\beta_{*}}, M \rightarrow \tilde{M} c_{d}^{\alpha_{*}}$. Consistency with (2.28) in the limit $M \rightarrow 0, F \rightarrow 1+\Gamma M^{2 / 3}$ requires that $5 \alpha_{*} / 3+\beta_{*} / 2=1$. This leaves an arbitrary choice for $\alpha_{*}$, and the value giving the best fit empirically is found to be $\alpha_{*}=2 / 5$ yielding, since the drag scales with $M^{5 / 3}$,

$$
\mathscr{D}_{m} \approx k c_{d}^{2 / 3}
$$

with $k$ a constant which can be estimated from a least-squares fit for the five obstacles to be 1.156. $D_{m}$ is plotted against $k c_{d}^{2 / 3}$ in figure $2(b)$, and the extent to which the rescaled drag curves collapse to a single curve is shown in the inset. It is to be emphasized that the above relation is entirely empirical and has not been tested for obstacles other than for the five under discussion. Nevertheless, it may prove to be of use in understanding and developing drag parameterizations for oceanic and atmospheric orography.

In figure $2(c, d)$ the particular cases of the 'Witch of Agnesi' and the paraboloid are considered. In each case, the drag curve $D(\Gamma)$ is plotted along with the drag $\mathscr{D}$, scaled by $M^{5 / 3}$, calculated from the corresponding shallow-water results with $M=0.05,0.1$ and 0.4. For the shallow-water results $\Gamma$ is the transcritical similarity parameter $\Gamma=(F-1) M^{-2 / 3}$. These plots allow the accuracy of the transcritical theory to be assessed. It is interesting to note that the shallow-water results diverge from the TSD results more rapidly for subcritical flow $(\Gamma<0)$ than for supercritical flow $(\Gamma>0)$. For $M \lesssim 0.4$ the transcritical theory accurately predicts the peak drag, which occurs in the shallow-water flow for $\Gamma \approx 0.7-0.9$, to within an accuracy of $10 \%$. On the subcritical side, however, the prediction is in error, by as much as $50 \%$ in the case of the paraboloid.

Figure 3 illustrates the self-similarity of transcritical flows over the 'Witch of Agnesi' obstacle at constant values of $\Gamma$. The upper panels show the TSD flow around the equivalent aerofoil, the middle panels show the results for obstacle height $M=0.05$ and the lower panels show the results for $M=0.4$. Note that the $y$-axis in each 

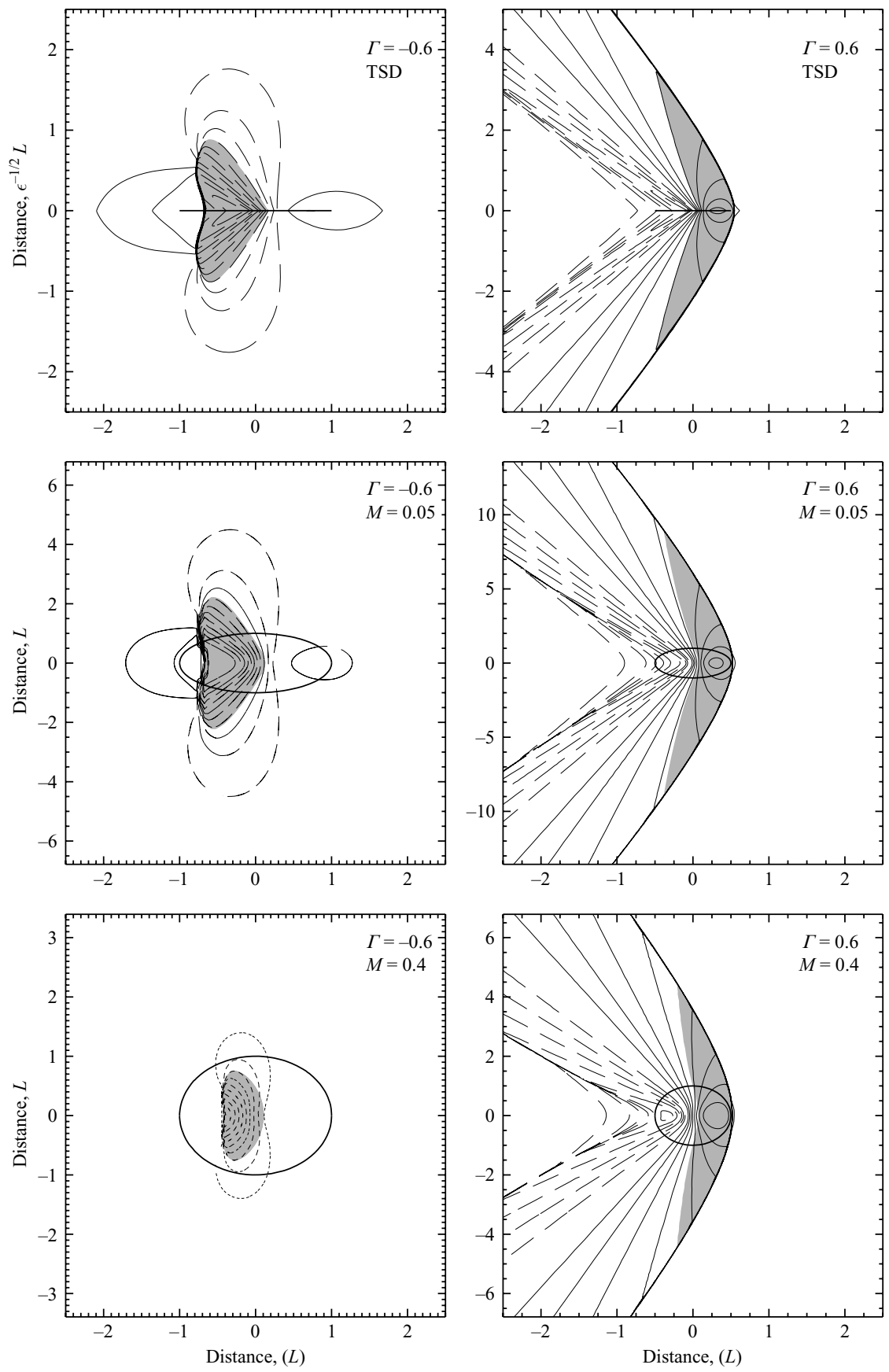

FIGURE 3. Steady-state height fields $\eta$ illustrating the transcritical similarity theory for flow over the 'Witch of Agnesi' obstacle. The upper panels show results derived from numerical solutions of the TSD equation ((2.19), with spatial resolution $\delta x=0.01 L$ and domain size $40 L \times$ $40 \epsilon^{-1 / 2} L$ ), and the remaining panels are derived from numerical solutions of the shallow-water equations $((2.4)$, with the following spatial resolutions and domain sizes: left, $\delta x=0.05 L$, $60 \mathrm{~L} \times 45 \mathrm{~L}$; right, $0.01 \mathrm{~L}, 10 \mathrm{~L} \times 10 \mathrm{~L}$ ) for obstacle heights $M=0.05$ and 0.4 . The left-hand panels show subcritical flow with transcritical similarity parameter $\Gamma=-0.6$, and the right-hand panels show supercritical flow with $\Gamma=0.6$. The contour intervals are $0.1 \epsilon H$ in each panel $\left(\epsilon=M^{2 / 3}\right)$, and the $y$-axis extends from $-2.5 \epsilon^{-1 / 2} L$ to $2.5 \epsilon^{-1 / 2} L$ (left panels) to $-5 \epsilon^{-1 / 2} L$ to $5 \epsilon^{-1 / 2} L$ (right panels). Regions of supercritical flow (left-hand panels) and subcritical flow (right-hand panels) are shaded. 
panel has been scaled by $\epsilon^{1 / 2}=M^{1 / 3}$, and the contour intervals have been scaled by $\epsilon$ for consistency with the transcritical asymptotic theory. Hence the obstacle, where plotted, appears elliptical in the lower panels. In the left-hand panels the oncoming flow is subcritical $(\Gamma=-0.6)$, and the shaded region indicates an isolated region of supercritical flow, beginning ahead of the obstacle (or aerofoil) and extending to a hydraulic jump at its rear. In the right-hand panels the oncoming flow is supercritical $(\Gamma=0.6)$, and the shading indicates a region of subcritical flow. At the upstream boundary of the subcritical region is a hydraulic jump ahead of which the flow is undisturbed. For both the subcritical and supercritical oncoming flows, the TSD and $M=0.05$ pictures appear nearly identical, but for the $M=0.4$ case some differences are apparent as the transcritical asymptotic theory becomes inaccurate. The differences in the flow patterns are much more pronounced for the subcritical flows, because in subcritical flow the disturbance is concentrated in a much smaller region in the vicinity of the obstacle. As $M$ increases, the overlap between the 'inner' flow region, where the flow passes over the obstacle, and the 'outer' flow region away from the obstacle is much greater for subcritical flow. Therefore, for subcritical flow one of the key assumptions of the transcritical theory becomes invalid at lower values of $M$, as is also apparent in figure 2 .

Figure 4 is a plot of the drag $\mathscr{D}$, scaled this time by $M^{2}$, against the Froude number $F$ of the oncoming flow. The upper panel shows the results for the 'Witch of Agnesi' obstacle and the lower panel the results for the paraboloid. The numerical results have been replotted with this scaling to emphasize the different regions of validity of the transcritical theory and the supercritical theory. The dashed curve in each picture is the linear supercritical drag (2.28), and the series of solid curves shows the TSD prediction for the transcritical drag, as previously plotted in figure 2, for the obstacle heights $M=0.05,0.1$ and 0.4. These TSD drag curves are plotted for $\Gamma_{-}<\Gamma<1.5$ in each case, with $\Gamma_{+}$illustrated as a solid point. The shallow-water numerical results for obstacle heights $M=0.05,0.1$ and 0.4 are plotted as symbols (triangles, diamonds and squares respectively). It is clear that for each obstacle and for $M=0.05$ and 0.1 in particular, the calculated drags follow the TSD prediction for Froude numbers $F$ satisfying $\Gamma_{-}<\Gamma \lesssim \Gamma_{+}$. For $F$ satisfying $\Gamma \sim \Gamma_{+}$, the calculated drags have begun to diverge from the TSD prediction and rapidly converge to the supercritical linear prediction given by the dashed curve, which is accurate for values of $F$ corresponding to $\Gamma \gtrsim 1.5$. These results indicate that the transition region between the realms of validity of the transcritical and supercritical asymptotic theories occurs rapidly and that the transcritical and supercritical theories together constitute a complete description of the behaviour observed at obstacle heights up to $M=0.4$. For $M=0.4$, a similar picture is evident for each obstacle, except that it is clear that (as seen previously in figure 2) the transcritical-theory prediction is less accurate for subcritical oncoming flows.

Lamb \& Britter (1984); Schär \& Smith (1993a); Baines (1995) and Jiang \& Smith (2000) used experimental and numerical results to construct regime diagrams for flow over three-dimensional obstacles. The shallow-water finite-volume numerical code allows for accurate calculation of the boundaries between different flow regimes. In particular, the accuracy of the predictions (2.21) for the boundaries between transcritical and subcritical flow and between transcritical and supercritical flow can be tested. In the TSD problem of flow over a thin aerofoil, the boundary between the transcritical and supercritical flow regimes is easily calculated by varying the parameter $\Gamma$ until the subcritical region near the obstacle vanishes. In shallow-water flow over the corresponding axisymmetric obstacle the situation can sometimes 

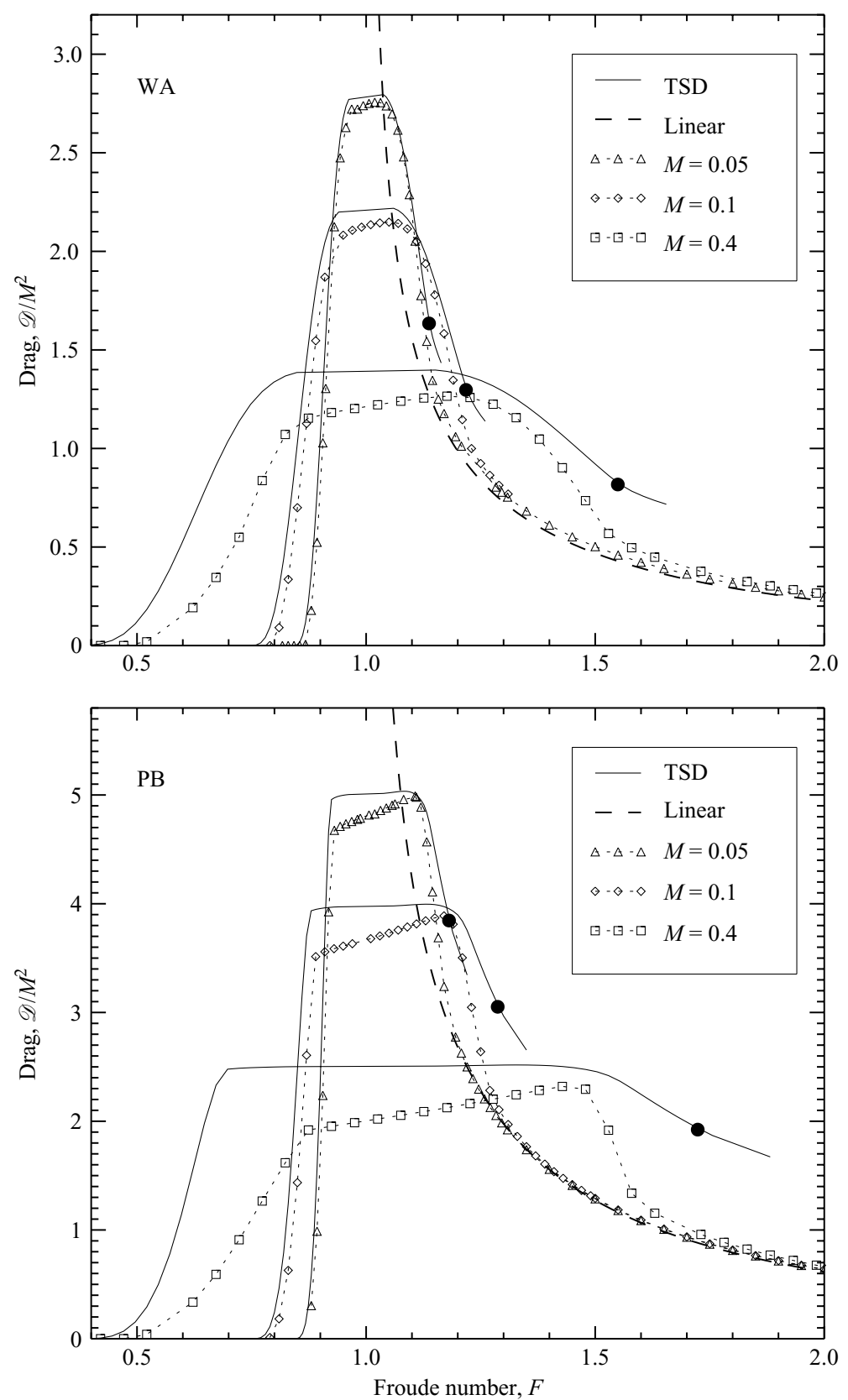

FIGURE 4. The drag $\mathscr{D}$, scaled by the obstacle height $M^{2}$, as a function of Froude number $F$ for flow over the 'Witch of Agnesi' obstacle (WA) and the paraboloid obstacle (PB) (see table 1). The triangles, diamonds and squares mark the shallow-water-model results for $M=0.05,0.1$ and 0.4 , respectively. The solid lines show the transcritical-similarity-theory predictions for the drag in each case, as derived from the TSD numerical results (see also figure 2); the Froude numbers corresponding to $\Gamma=\Gamma_{+}$are marked as solid circles. The dashed curve shows the drag in linear supercritical flow as given by (2.28). 

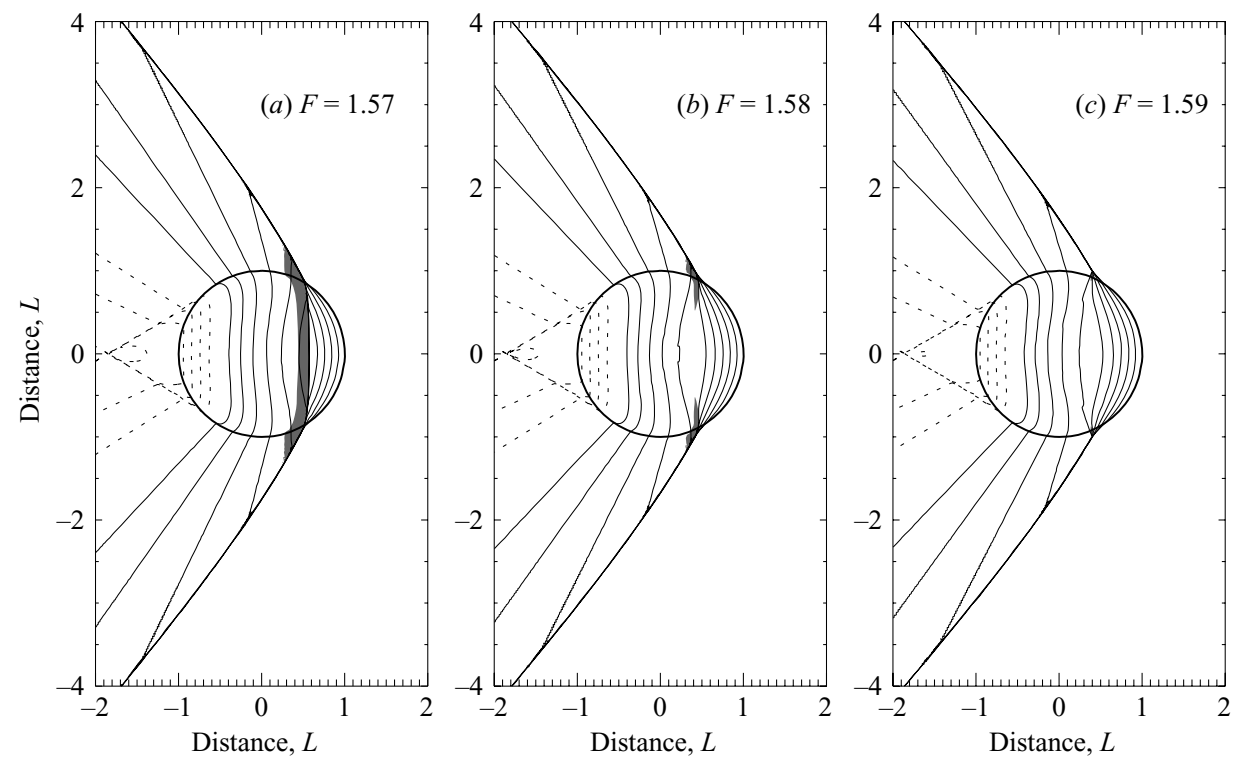

FIGURE 5. The steady-state free-surface displacement $(\eta=\sigma-1+M h)$ calculated from numerical solutions of the shallow-water equations (2.4). The plots illustrate the transition, occurring for flow over the paraboloid obstacle with $M=0.4$, between ( $a$ a $F=1.57)$ supercritical flow with a single embedded region of subcritical flow (shaded), ((b) $F=1.58)$ two separate regions of subcritical flow and $((c) F=1.59)$ purely supercritical flow. The contour interval is $0.1 \mathrm{H}$ in each panel, the dashed curves indicating negative surface displacements; the zero contour is omitted.

be more complicated, as the isolated region of subcritical flow can split into two regions, located on the flanks of the obstacle. This type of supercritical transition, which occurs for the paraboloid (but not for the 'Witch of Agnesi'), is illustrated in figure 5. The three panels show the steady-flow height field for $M=0.4$ and $F=1.57,1.58$ and 1.59 respectively. At $F=1.57$ a single 'bow' hydraulic jump is situated ahead of the obstacle (Jiang \& Smith 2000), behind which is a region of subcritical flow (shaded). As $F$ increases to 1.58 the bow jump at the centreline $y=0$ weakens and then disappears, and the subcritical region divides into two regions located at the edge of the obstacle (middle panel). As $F$ increases further to $F=1.59$, the two subcritical regions reduce in size and then disappear entirely. By $F=1.59$ (right-hand panel) the bow jump has divided into two entirely supercritical 'flank' jumps to the sides of the obstacle. For the paraboloid obstacle, then, there are two separate transition points, the first when the flow becomes supercritical everywhere along the centreline of the obstacle and the second when the flow becomes supercritical everywhere in the domain. The existence of these two separate transition points may raise doubts concerning the validity of hydraulic theories derived from the behaviour of the flow along the centre streamline (see e.g. Baines 1995, pp. 82-88). However, further numerical results indicate that the parameter values of the transitions remain close together even for larger obstacle heights (results not shown).

Figure 6 shows the calculated regime diagrams for both the 'Witch of Agnesi' and the paraboloid. The region of parameter space where entirely supercritical flow is found is labelled SPC, that with subcritical flow SBC and that with transcritical flow TC. Note that the transcritical theory of $\S 2$ becomes formally accurate as $M \rightarrow 0, F \rightarrow 1$, whilst remaining within the transcritical (TC) region of figure 6 , and 

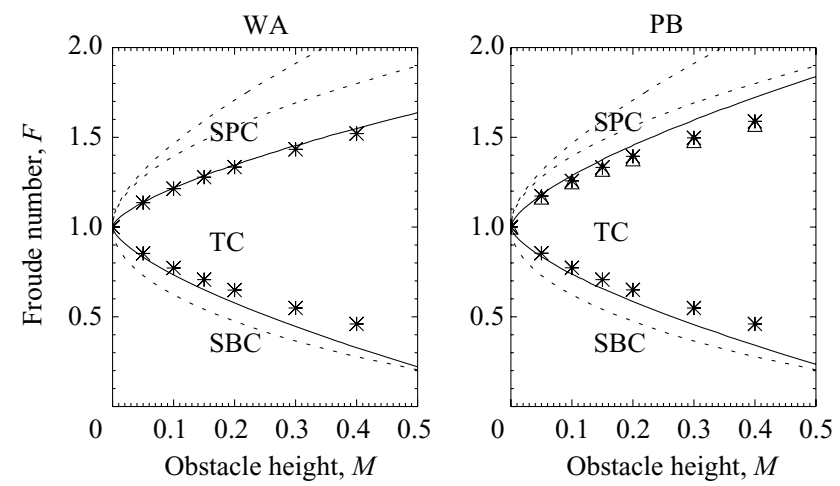

FIGURE 6. Illustrating the flow regimes (SBC, subcritical; TC, transcritical; SPC, supercritical), in obstacle-height Froude-number $(M, F)$ parameter space, for flow over the 'Witch of Agnesi' (WA) and paraboloid (PB) obstacles. The solid lines show the predictions (2.21) from transcritical theory, formally valid for $M \ll 1$. The dotted curves show the corresponding results for two-dimensional obstacles, valid for all $M$ (see e.g. Baines 1995; note that there are two curves on the supercritical side, since a region of hysteresis exists). The stars show the location of the actual transitions found in each case from a sequence of steady numerical solutions of the shallow-water equations (2.4). The triangles in the paraboloid panel mark the transition from a single subcritical region to two distinct subcritical regions, as illustrated in figure 5. Empirical regime diagrams, including aspects such as wake stability and penetration of the fluid-layer surface by the obstacle, are given in Baines (1995) and Jiang \& Smith (2000), without the solid lines given by the theory presented here.

is approximately accurate throughout the entire TC region shown; the supercritical theory, however, is formally accurate as $M \rightarrow 0$ within the supercritical (SPC) region and is approximately valid everywhere in the SPC region away from the transition boundary. The introduction of dispersion (see the following subsection) does not greatly alter these regions of validity. The locations in $(M, F)$-space where transitions have been found to occur in the numerical calculations are labelled on figure 6 with stars, whereas diamonds mark the locations where the subcritical region is found to divide into two in the paraboloid obstacle flows. The predictions from the transcritical theory (2.21), expected to be accurate for small $M$, are plotted as a solid curve. The dotted curves show the corresponding results, valid for all $M$, for two-dimensional obstacles (see e.g. Baines 1995; note that there are two curves on the supercritical side, as a region of hysteresis exists). The calculated locations of the transition curves (the stars) diverge somewhat from the transcritical theory (the solid curves) for values of $M$ approaching 0.5 , although this is perhaps not surprising as the transcritical theory has been shown above to be less accurate when $|\Gamma|$ is relatively large, as is in the vicinity of the transition curves. Other interesting flow regimes, exist for $M>0.5$. For example, in subcritical oncoming flow the obstacle wakes are known to become unstable (Schär \& Smith 1993b), and at higher obstacle heights there is the possibility of 'drying out' when the surface of the obstacle penetrates through the top of the fluid layer (Schär \& Smith 1993a; Jiang \& Smith 2000).

In summary, the transcritical equivalent-aerofoil theory, formally valid for small obstacle height $(M \ll 1)$, describes most aspects of the full shallow-water flow accurately up to $M=0.4$, particularly the peak drag experienced by the obstacle, which typically occurs for supercritical oncoming flow $\Gamma \sim 0.7$. For certain aspects, however, particularly the flow patterns and drag for subcritical oncoming flow and the prediction of the exact locations of supercritical and subcritical transitions in 
parameter space, the transcritical predictions diverge from the shallow-water results even at comparatively low obstacle heights. Nevertheless, the transcritical theory appears to provide a robust way of comparing the effect of different axisymmetric obstacles on the flow, and in particular the empirical formula (3.1) may be of use as a practical method of estimating the peak drag exerted by obstacles on the flow for a wide range of obstacles.

\subsection{Weakly dispersive transcritical flow}

How do weakly dispersive transcritical flows over axisymmetric obstacles differ from non-dispersive flows? In flow over two-dimensional topography it is well known (e.g. Grimshaw \& Smyth 1986) that instead of a single upstream-propagating hydraulic jump as in non-dispersive flow, an upstream-propagating cnoidal wavetrain is present in the corresponding dispersive flow. The drag over the obstacle then varies in time with the period of the cnoidal wavetrain. This raises the question whether the drag is steady or unsteady in the case of flow over a three-dimensional obstacle. Does two-dimensionality inhibit the upstream propagation of any nonlinear wavetrain that is generated? How do the positions of nonlinear wavefronts relate to the positions of hydraulic jumps in the corresponding non-dispersive flow? Some of these questions were addressed by Johnson \& Vilenski (2004), who considered flow over a quasi-threedimensional obstacle, i.e. an obstacle elongated asymptotically in the cross-stream direction. In contrast to the previous work, here we use the boundary conditions derived from the equivalent aerofoil of the obstacles (2.18) to force the KP equation (2.15) in order to find solutions consistent with flow over an axisymmetric three-dimensional obstacle. The weakly dispersive results do not differ qualitatively from those of Johnson \& Vilenski (2004) or from those for flows forced from a moving pressure distribution (Katsis \& Akylas 1987; Lee \& Grimshaw 1990) but are of particular interest in that they allow the results for weakly dispersive and non-dispersive flows to be compared directly, by examining the results from the TSD equation (2.19) under identical forcing. Also, unlike the results of Johnson \& Vilenski (2004) the current results can be definitively associated with the flow over a specific physical obstacle (in Johnson \& Vilenski (2004) the obstacle's cross-stream dimension varies as $\epsilon^{-1 / 2}$ ).

Figure 7 shows for comparison the steady height fields for non-dispersive and weakly dispersive transcritical flows past the equivalent aerofoil corresponding to the paraboloid obstacle. The upper panels are derived from steady solutions of (2.19) at $\Gamma=-0.3$ and 0.5 , representing the situation for typical subcritical and supercritical oncoming flows, respectively. The lower panels show the corresponding steady solutions of (2.15) with $\Delta=0.5$ and 0.25 . Regions of supercritical flow, defined by $\eta_{0}^{o}<2 \Gamma / 3$, are shaded in the left-hand panels, and regions of subcritical flow, for which $\eta_{0}^{o}>2 \Gamma / 3$, are shaded in the right-hand panels. Considering first the case of subcritical oncoming flow $(\Gamma=-0.3)$, the non-dispersive flow has a region of supercritical flow located to the rear of the equivalent aerofoil, followed by a characteristic 'fishtail' system of hydraulic jumps. The corresponding dispersive flows bear little resemblance to the non-dispersive flow. As expected from linear theory, a wavetrain of dispersive waves is generated downstream of the obstacle. The wavetrain has sufficient amplitude that the flow oscillates from subcritical to supercritical within the phase of each wave, although the wavetrain itself exhibits no apparent sign of nonlinearity as a consequence. Upstream of the obstacle's equivalent aerofoil there is a relatively weak wavetrain of dispersive waves.

In the case of supercritical oncoming flow (shown in the panels with $\Gamma=0.5$ ), there are some striking similarities between the non-dispersive and dispersive height fields. 

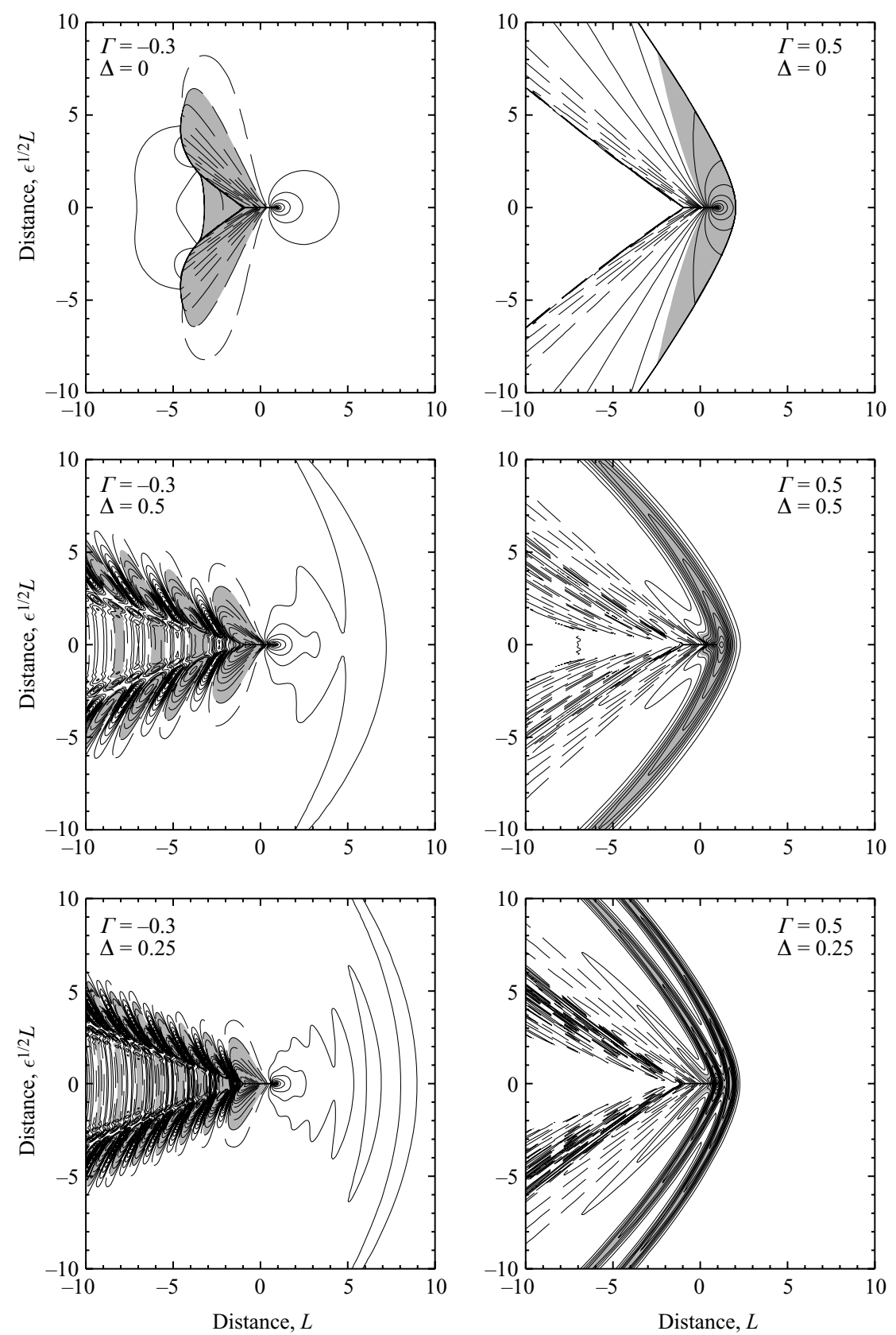

FIGURE 7. Non-dispersive and dispersive transcritical height-field patterns $\eta_{0}^{o}(x, Y)$ for steady flow around the paraboloid obstacle, in the transcritical limit. The top panels show the height field derived from numerical solutions of the non-dispersive TSD equation (2.19) for $\Gamma=-0.3$ (left) and $\Gamma=0.5$ (right). The remaining panels show the corresponding results obtained from numerical solutions of the dispersive KP equation (2.15) for two different values of the dispersion parameter $(0.5$ and 0.25$)$. The contour interval is $0.1 \epsilon H$ in each panel, negative contours are dashed and the zero contour is omitted. The shaded regions in the left-hand panels correspond to regions of supercritical flow, whereas in the right-hand panels the shading corresponds to regions of subcritical flow. 


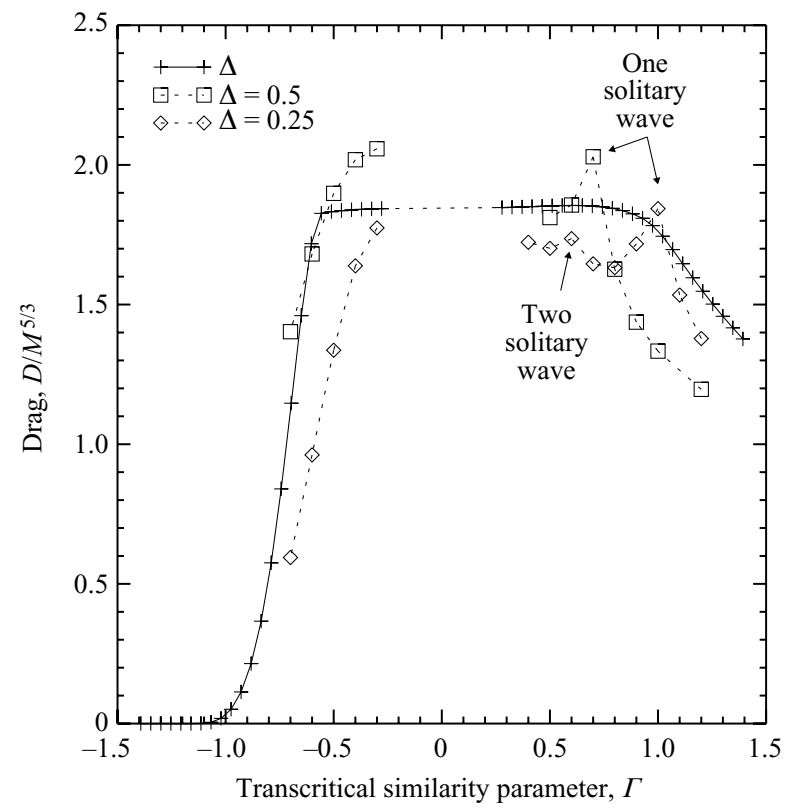

FiguRE 8 . The steady transcritical drag function $D(\Gamma)$ vs. the transcritical similarity parameter $\Gamma$ for the paraboloid obstacle, for non-dispersive flow $(\Delta=0)$ and weakly dispersive flows with $\Delta=0.5$ ) and $\Delta=0.25$, respectively. The non-dispersive and dispersive result were calculated from steady numerical solutions of (2.19) and locally steady solutions of (2.15), respectively.

At the location of the steady upstream hydraulic jump in the non-dispersive flow, there is a large-amplitude solitary wave in the dispersive flow with $\Delta=0.5$. In the dispersive flow with $\Delta=0.25$ this single solitary wave is replaced by a pair of separate solitary waves. In the non-dispersive flow the height field decreases uniformly behind the upstream jump, eventually becoming negative and then returning at a downstream jump (the 'V-wave') to the undisturbed height. In the dispersive flows, the height field remains nearly constant in a relatively large region behind the initial solitary wave(s). Linear wavetrains spread out dispersively in the region behind the constant-heightfield region, and the lines tracing out the centre of these wavetrains are almost exactly coincident with the location of the downstream V-wave in the non-dispersive flow.

Figure 8 shows for comparison the drag function $D(\Gamma)$ for non-dispersive flow and weakly dispersive flow with $\Delta=0.25$ and 0.5 for the paraboloid obstacle. For the dispersive flows, the drags plotted are the mean drags obtained for $\tau=10-12$ (small domain) or $\tau=20-22$ (large domain) for those flows where the rate of change of the height field in the immediate vicinity of the obstacle satisfied a steady-state criterion. For flows with $\Gamma$ in the range $[-0.2,0.4]$ it was not found to be possible to satisfy the steady state criterion using either of the chosen domains. In figure 8 the drag can be seen to remain remarkably constant between each of the three scenarios. The subcritical drag is increased slightly as dispersion increases, as is consistent with the increasing amplitude of the wavetrains generated to the rear of the obstacle. For supercritical oncoming flow $(\Gamma>0)$ the drag oscillates as a function of $\Gamma$ as solitary waves form and move ahead of the obstacle. As found by Johnson \& Vilenski (2004), the maxima in the drag correspond to situations with one or two distinct largeamplitude solitary waves ahead of the obstacle, and the minima to the intermediate situation. High values of drag are sustained for larger values of $\Gamma$ when dispersion is 
weaker: in this case at least one solitary wave exists ahead of the obstacle for larger values of $\Gamma$.

\subsection{Non-dispersive supercritical flow}

For small $M$ in non-dispersive supercritical flow, the steady-flow pattern away from the obstacle for $y>0$ is described by the wake equation $(2.31)$ with $\bar{\Delta}=0$, i.e. a Hopf equation. The 'initial' condition at $\mathscr{Y}_{*}=0$ is given by the linear far-field solution (2.32). Whitham (1974) discussed the nature of the solution as $\mathscr{Y}_{*}$ increases away from the obstacle. Solutions of the Hopf equation $((2.31)$, with $\bar{\Delta}=0)$ become multivalued and must be regularized by hydraulic jumps, which, in order to conserve mass and momentum in the flow, must obey an equal-areas rule (see pp. 39-53 of Whitham 1974). Regardless of the exact form of the topographic initial condition, far enough from the obstacle the solution develops into an $N$-wave, in which the height field is linear between two hydraulic jumps. For an antisymmetric initial condition such as (2.32), the $N$-wave is centred on the Mach line $\mathscr{X}=0$ and, as $\mathscr{Y} *$ increases, the distance of each jump from the Mach line increases uniformly. The $N$-wave asymptotic solution for the current problem is given by

$$
\eta_{0}^{f}\left(\mathscr{X}, \mathscr{Y}_{*}\right) \rightarrow\left\{\begin{array}{ll}
\frac{2 \gamma}{3} \frac{\mathscr{X}}{\mathscr{Y}_{*}} & \text { for }|\mathscr{X}| \leqslant \sqrt{\frac{3 A \mathscr{Y}_{*}}{F^{2}-1}} \\
0 & \text { for }|\mathscr{X}|>\sqrt{\frac{3 A \mathscr{Y}_{*}}{F^{2}-1}}
\end{array} \quad \text { with } A=-\int_{0}^{\infty} \mathscr{X} G(\mathscr{X}) \mathrm{d} \mathscr{X} .\right.
$$

The parameter $A$ is a measure of both the extent and the amplitude of the $N$-wave at a fixed distance far from the obstacle. The values for $A$ for the obstacles in table 1 are $1 / 2, \sqrt{\pi / 8}, \sqrt{3 / 8}, \pi \sqrt{3} / 8,2 / 3$ for the 'Witch of Agnesi', Gaussian, cone, hemi-ellipsoid and paraboloid obstacles, respectively. These values of $A$ do not differ greatly, i.e. the amplitude of the $N$-wave far from the obstacle is relatively insensitive to the obstacle shape, for obstacles with the same volume. This is in contrast with the drag exerted by the obstacle on the flow in the supercritical regime, as seen in table 1.

Figure 9 shows the height field from a shallow-water calculation for the 'Witch of Agnesi' obstacle with $M=0.1, F=1.3$. The transcritical similarity parameter is therefore $\Gamma=1.392>\Gamma_{+}=1.012$ for this obstacle. Hence supercritical theory might be expected to be accurate for this flow. The solid dots marked on the figure show the Whitham prediction (3.2) for the position of the hydraulic jumps marking the boundary of the $N$-wave. Whitham's formula, which predicts that the jumps should have a parabolic profile centred on the Mach line, is seen to be accurate even close to the obstacle. It is notable that $\Gamma$ is not dramatically larger than $\Gamma_{+}$, at which the flow might be expected to switch between the transcritical and supercritical regimes, yet the supercritical theory is accurate. This result reinforces the impression from figure 4 that the region of parameter space for which neither transcritical nor supercritical theory is accurate is relatively small.

\subsection{Weakly dispersive supercritical flow}

In dispersive supercritical flow the wake development with increasing distance $\mathscr{Y}_{*}$ from the obstacle is determined by the $\mathrm{KdV}$ equation (2.31) with the initial condition at $\mathscr{Y}_{*}=0$ given by $(2.32)$ as in the non-dispersive case. Equation (2.31) together with (2.32) can be rescaled into the canonical problem

$$
u_{\bar{t}}-6 u u_{\mathscr{X}}+u_{\mathscr{X} X X}=0 \quad \text { with } u(\mathscr{X}, 0)=\mathscr{A} \mathscr{X} G(\mathscr{X}),
$$




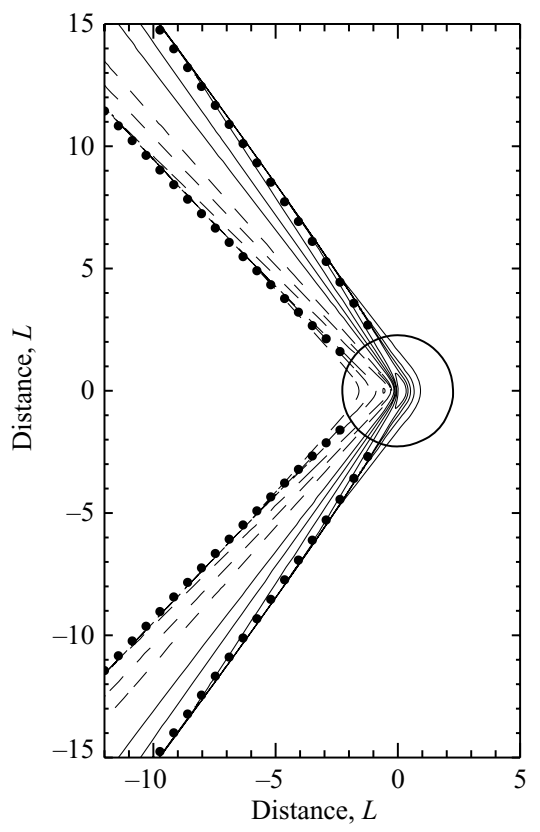

FiguRE 9. Steady-state free surface displacement $(\eta=\sigma-1+M h)$ flow over the 'Witch of Agnesi' obstacle with $M=0.1, F=1.3$. The contour interval is $0.1 H$, with positive displacements given by solid contours and negative displacements by dashed contours. The zero contour is omitted. The solid dots show the Whitham prediction (3.2) for the location of the hydraulic jumps associated with the front and rear of the $N$-wave.

the initial-condition amplitude $\mathscr{A}$ being given by

$$
\mathscr{A}=\frac{3 M}{2 \delta^{2} \sqrt{F^{2}-1}} .
$$

Note that $\mathscr{A}$ can be arbitrarily large without invalidating any assumption of the theory of $\S 2.3$, as $\delta$ may be arbitrarily small. Clearly, from (3.3), for a given obstacle the supercritical obstacle wake is self-similar to the extent that its qualitative nature is a function solely of the non-dimensional parameter $\mathscr{A}$. For each obstacle, (3.3) can be solved using the inverse scattering technique to obtain the exact solution for the wake.

One fundamental question concerns the number of distinct solitary waves that will emerge in the far field. To obtain this number as a function of $\mathscr{A}$ it is necessary only to determine the number of discrete positive eigenvalues $\kappa$ of the scattering problem

$$
\frac{\mathrm{d}^{2} \psi}{\mathrm{d} \mathscr{X}^{2}}-(\kappa-u(\mathscr{X}, 0)) \psi=0 \quad \text { with } \psi \rightarrow 0 \text { as } \mathscr{X} \rightarrow \pm \infty
$$

(e.g. Drazin \& Johnson 1988). This eigenvalue problem may be solved numerically using standard techniques. In practice, it is solved iteratively on a finite grid $-L<x<L$, where $L$ is chosen to be sufficiently large that $u( \pm L, 0) \approx 0$. To obtain an estimate $\kappa_{i}$ for a specific eigenvalue $\kappa$ at the $i$ th iteration, the boundary conditions at $\mathscr{X}= \pm \infty$ are replaced by $\psi_{\mathscr{X}}=\mp \sqrt{\kappa_{i-1}} \psi$ at $\mathscr{X}= \pm L$, where $\kappa_{i-1}$ is the previous estimate for $\kappa$. Numerical results have been tested by comparison with the analytic solution found for the hemi-ellipsoid obstacle presented in the Appendix, with agreement obtained up to four significant figures. 


\begin{tabular}{lccccccc}
\hline Obstacle & $\mathscr{D}_{m}$ & $\Gamma_{-}$ & $\Gamma_{+}$ & $\mathscr{A}_{1}$ & $\mathscr{A}_{2}$ & $\mathscr{A}_{3}$ & $\mathscr{A}_{4}$ \\
Witch of Agnesi & 1.030 & -1.236 & 1.012 & 13.87 & 34.09 & 62.56 & 99.57 \\
Gaussian & 1.504 & -1.225 & 1.100 & 20.76 & 61.86 & 124.0 & 207.1 \\
cone & 1.411 & -1.237 & 1.034 & 22.51 & 71.68 & 148.3 & 252.3 \\
hemi-ellipsoid & 2.356 & -1.208 & 1.739 & 29.09 & 95.06 & 198.7 & 340.1 \\
paraboloid & 1.810 & -1.214 & 1.333 & 26.24 & 83.76 & 173.3 & 294.8
\end{tabular}

TABLE 2. Numerically calculated values of the maximum drag coefficient $\mathscr{D}_{m}$ for each obstacle in the transcritical regime $\left(\max \mathscr{D}=\mathscr{D}_{m} M^{5 / 3}\right)$, and values of the transcritical similarity parameter marking the boundary between subcritical and transcritical flow $\left(\Gamma_{-}\right)$and supercritical and transcritical flow $\left(\Gamma_{+}\right)$. Columns $5-8$ give the values of $\mathscr{A}=3 M /\left(2 \delta^{2} \sqrt{F^{2}-1}\right)$ separating different solitary-wave regimes $\left(\mathscr{A}<\mathscr{A}_{1}\right.$, one solitary wave; $\mathscr{A}_{1}<\mathscr{A}<\mathscr{A}_{2}$, two solitary waves; etc.) in the dispersive supercritical wakes of each obstacle. The values were obtained by numerical solution of the scattering eigenvalue problem (3.5) except in the case of the hemi-ellipsoid, for which an analytic solution is presented in Appendix A. The results are accurate to the four significant figures given, on the basis of the convergence properties of the hemi-ellipsoid numerical results.

Table 2 shows for each obstacle those specific values of $\mathscr{A}$ that mark the boundaries between the single-solitary-wave regime $\left(\mathscr{A}<\mathscr{A}_{1}\right)$, the two-solitary-wave regime $\left(\mathscr{A}_{1}<\mathscr{A}<\mathscr{A}_{2}\right)$, the three-solitary-wave regime etc. It is clear that the dispersive supercritical wakes of the less compact obstacles (particularly that of the Witch of Agnesi) tend to break up into more solitary waves at lower values of $\mathscr{A}$ (i.e. lower obstacle heights) when compared with the supercritical wakes of more compact obstacles (the paraboloid or the hemi-ellipsoid). This is consistent with the fact that broader potentials, such as those derived from the wakes of the less compact obstacles, tend to have more discrete eigenvalues, each corresponding to a trapped eigenfunction, for a given amplitude $\mathscr{A}$.

\section{Conclusions}

The main contribution of this work has been to describe explicitly a similarity theory for transcritical single-layer flow over a small isolated obstacle in terms of a transcritical similarity parameter $\Gamma=(F-1) / M^{2 / 3}$. The problem has been found to be isomorphic to that of transonic flow over a thin 'equivalent aerofoil' with thickness proportional to the cross-sectional area of the obstacle. The utility and limitations of the theory, for both non-dispersive and weakly dispersive flow, have been explored numerically. In particular, the theory is found to be inaccurate for $\Gamma \gtrsim 1.5$, and a supercritical theory in which the flow is linear in the vicinity of the obstacle is found to apply in its place. The influence of the obstacle shape on the drag exerted by the obstacle on the flow has been described in the transcritical and supercritical regimes; empirically, there is a surprisingly close relationship, (3.1), which may merit further investigation and may offer some insight of use in the development of parameterization schemes for ocean circulation, climate and numerical weather-prediction models.

The current work also gives some insight into the similarities and differences between two-dimensional weakly dispersive and non-dispersive transcritical and supercritical flows. Historically, the non-dispersive shallow-water model has been used to develop important paradigms of many laboratory and geophysical flow phenomena. However, modern observational techniques, such as synthetic-aperture radar (e.g. Li et al. 2004), raise the possibility that many mesoscale phenomena in 
the atmosphere and oceans are strongly dispersive in nature. Weakly nonlinear model equations, such as the KP equation (2.15), and their strongly nonlinear counterparts (Green \& Naghdi 1976; Choi \& Camassa 1999), are likely to be of great value in understanding and interpreting such observations, as well as in the development of parameterizations for general circulation models.

Of considerable interest is the relationship between the current results and those for stratified flows over three-dimensional topography. There is no obstacle to deriving the transcritical forced KP equation (2.15), following Grimshaw \& Smyth (1986), who consider flow over a two-dimensional obstacle for the case of stratified flow in the presence of a reflecting upper boundary. In the stratified case critical flow, corresponding to $\Gamma=0$ in (2.15), occurs when a vertical normal mode of the system becomes resonant to long-wave forcing. In practical situations, whether in the laboratory, ocean or atmosphere, the most physically relevant mode is likely to be the gravest mode, for which direct measurements of amplitude may be possible. A further special case of interest is that of Boussinesq flow with two approximately equal layers of different densities. In this particular physical situation, the scaling in the derivation of (2.15) is necessarily altered and a cubic nonlinearity enters (e.g. Grimshaw, Chan \& Chow 2002). The effects of rotation and obstacle anisotropy also merit further investigation.

E.R.J. and O.J.R. acknowledge funding from the UK Natural Environment Research Council through grant no. NER/A/S/2000/01323 and research studentship NER/S/A/2003/11387.

\section{Appendix. The scattering problem for a hemi-ellipsoid}

Here an analytic equation is derived for the eigenvalues $\kappa$ of the scattering problem (3.5) for the case of a hemi-ellipsoid obstacle. The reason that the hemi-ellipsoid case is straightforward is that, from table 1 , the potential $u(\mathscr{X}, 0)$ takes a simple form,

$$
u(\mathscr{X}, 0)=\mathscr{A} \mathscr{X} G(\mathscr{X})=\mathscr{A} \begin{cases}-\beta \mathscr{X}, & |\mathscr{X}| \leqslant a, \\ 0, & |\mathscr{X}|>a,\end{cases}
$$

where $\beta=\pi / 2 a$ and the normalisation constant $a=\sqrt{3} / 2$ as given in table 1 . Hence the scattering equation (3.5) has a general solution

$\psi(\mathscr{X})= \begin{cases}\alpha_{1} \exp \{\sqrt{\kappa} \mathscr{X}\}, & \mathscr{X}<-a, \\ \alpha_{2} \operatorname{Ai}\left(\mathscr{A}^{-2 / 3} \mathscr{B}^{-2 / 3}(\kappa+\mathscr{A} \mathscr{B} \mathscr{X})\right)+\alpha_{3} B i\left(\mathscr{A}^{-2 / 3} \mathscr{B}^{-2 / 3}(\kappa+\mathscr{A} \mathscr{B} \mathscr{X})\right), & |\mathscr{X}| \leqslant a, \\ \alpha_{4} \exp \{-\sqrt{\kappa} \mathscr{X}\}, & \mathscr{X}>a,\end{cases}$

for constants $\alpha_{1}-\alpha_{4}$. Ai and Bi are Airy functions. Applying the matching conditions that $\psi, \psi_{\mathscr{X}}$ are continuous at $x= \pm a$, an eigenvalue equation for $\kappa$ is obtained,

$$
\begin{aligned}
& {\left[\operatorname{Ai}^{\prime}(\tilde{\kappa}-p)-\sqrt{\tilde{\kappa}} \operatorname{Ai}(\tilde{\kappa}-p)\right]\left[\operatorname{Bi}^{\prime}(\tilde{\kappa}+p)+\sqrt{\tilde{\kappa}} \operatorname{Bi}(\tilde{\kappa}+p)\right]} \\
& =\left[\operatorname{Ai}^{\prime}(\tilde{\kappa}+p)-\sqrt{\tilde{\kappa}} \operatorname{Ai}(\tilde{\kappa}+p)\right]\left[\operatorname{Bi}^{\prime}(\tilde{\kappa}-p)-\sqrt{\tilde{\kappa}} \operatorname{Bi}(\tilde{\kappa}-p)\right]
\end{aligned}
$$

where $\tilde{\kappa}=\kappa \mathscr{A}^{-2 / 3} \beta^{-2 / 3}$ and $p=\beta^{1 / 3} \mathscr{A}^{1 / 3} a$. The values $\mathscr{A}_{1}, \mathscr{A}_{2}, \mathscr{A}_{3}, \ldots$ that mark the boundaries between the single-soliton, two-soliton and three-soliton etc. regimes may be determined by setting $\tilde{\kappa}=0$ in the above equation, and the roots of the resulting equation in $\mathscr{A}$ will then correspond to those values of $\mathscr{A}$ at which a new trapped 
eigenfunction with a positive eigenvalue emerges. This occurs at the roots of

$$
\operatorname{Ai}^{\prime}(p) \operatorname{Bi}^{\prime}(-p)=\operatorname{Ai}^{\prime}(-p) \operatorname{Bi}^{\prime}(p),
$$

which occur at $p_{i}=\{3.248,4.820,6.163,7.372, \ldots\}$, for which, inserting the values of $a=\sqrt{3} / 2, \beta=\pi / 2 a$ as above, we have $\mathscr{A}_{i}=\{29.09,95.06,198.7,340.1, \ldots\}$. These are the values of $\mathscr{A}$ presented for the hemi-ellipsoid obstacle in table 2 .

\section{REFERENCES}

Akylas, T. R. 1994 Three-dimensional long water-wave phenomena. Annu. Rev. Fluid Mech. 26, 191-210.

Badgley, P. C., Miloy, L. \& Childs, L. 1969 Oceans from Space. Gulf.

BaInes, P. G. 1995 Topographic Effects in Stratified Flows. Cambridge University Press.

BURK, S. D. \& HAACK, T. 1999 The dynamics of wave clouds upwind of coastal orography. Mon. Weath. Rev. 28, 1438-1455.

Chapman, C. J. 2000 High Speed Flow. Cambridge University Press.

Choi, W. \& Camassa, R. 1999 Fully nonlinear internal waves in a two-fluid system. J. Fluid Mech. 396, 1-36.

Cole, J. D. \& Cook, P. 1986 Transonic Aerodynamics. North-Holland.

Drazin, P. G. \& Johnson, R. S. 1988 Solitons: An Introduction. Cambridge University Press.

ENGQuist, B. \& Osher, S. 1980 Stable and entropy satisfying approximations for transonic flow calculations. Math. Comput. 34, 45-75.

FornberG, B. \& Driscoll, T. A. 1999 A fast spectral algorithm for nonlinear wave equations with linear dispersion. J. Comput. Phys. 155, 456-467.

GoorJian, P. M. \& VAN Buskirk, R. 1981 Implicit calculations of transonic flows using monotone methods. AIAA Paper 1981-331.

Green, A. E. \& NAGHDI, P. M. 1976 A derivation of equations for wave propagation in water of variable depth. J. Fluid Mech. 78, 237-246.

Grimshaw, R. H. J., Chan, K. H. \& CHOw, K. W. 2002 Transcritical flow of a stratified fluid: The forced extended Korteweg-de Vries model. Phys. Fluids 14, 755-774.

Grimshaw, R. H. J. \& Melville, W. K. 1989 On the derivation of the modified KadomtsevPetviashvili equation. Stud. Appl. Maths 80, 183-203.

Grimshaw, R. H. J. \& Smyth, N. 1986 Resonant flow of a stratified fluid over topography. J. Fluid Mech. 169, 429-464.

Gurevich, A. V., Krylov, A. L., Khodorovsky, V. V. \& El, G. A. 1995 Supersonic flow past bodies in dispersive hydrodynamics. J. Expl Theor. Phys. 80, 87-96.

Gurevich, A. V., Krylov, A. L., Khodorovsky, V. V. \& El, G. A. 1996 Supersonic flow past finite-length bodies in dispersive hydrodynamics. J. Expl Theor. Phys. 82, 709-714.

JAMESON, A. 1979 Acceleration of transonic potential flow calculations on arbitrary meshes by the multiple grid method. AIAA Paper 1979-1458.

JiANG, Q. \& Sмiтh, R. B. 2000 V-waves, bow shocks, and wakes in supercritical hydrostatic flow. $J$. Fluid Mech. 406, 27-53.

Johnson, E. R., Esler, J. G., Rump, O. J., Sommeria, J. \& Vilenski, G. G. 2006 Orographically generated nonlinear waves in rotating and nonrotating two-layer flow. Proc. R. Soc. Lond. A 462, 3-20.

Johnson, E. R. \& Vilenski, G. G. 2004 Flow patterns and drag in near-critical flow over isolated orography. J. Atmos. Sci. 61, 2909-2918.

Kadomtsev, B. B. \& Petviashvili, V. I. 1970 On the stability of solitary waves in weakly dispersing media. Sov. Phys. Dokl. 15, 539-541.

Katsis, C. \& AkYLaS, T. R. 1987 On the excitation of long nonlinear water waves by a moving pressure distribution: Part 2. Three-dimensional effects. J. Fluid Mech. 177, 49-65.

Lamb, V. R. \& Britter, R. E. 1984 Shallow flow over an isolated obstacle. J. Fluid Mech. 147, 291-313.

LeE, S. J. \& Grimshaw, R. H. J. 1990 Upstream-advancing waves generated by 3-dimensional moving disturbances. Phys. Fluids A 2, 194-201. 
LeVeque, R. J. 2002 Finite Volume methods for Hyperbolic Problems. Cambridge University Press.

Li, X. F., Dong, C. M., Clemente-Colon, P., Pichel, W. G. \& Friedman, K. S. 2004 Synthetic aperture radar observation of the sea surface imprints of up- stream atmospheric solitons generated by flow impeded by an island. J. Geophys. Res. 209, C02016.

LI, Y. \& Sclavounos, P. D. 2002 Three-dimensional nonlinear solitary waves in shallow-water generated by an advancing disturbance. J. Fluid Mech. 470, 383-410.

Maxworthy, T., Dhieres, G. C. \& Didelle, H. 1984 The generation and propagation of internal gravity waves in a rotating fluid. J. Geophys. Res. 89, 6383.

MeI, C. C. 1976 Flow around a thin body moving in shallow-water. J. Fluid Mech. 77, 737-751.

MeI, C. C. 1989 The Applied Dynamics of Ocean Surface waves. World Scientific.

Murman, E. M. \& Cole, J. D. 1971 Calculations of plane steady transonic flows. AIAA J. 9, $114-121$.

RoE, P. L. 1981 Approximate Riemann solvers, parameter vectors, and difference schemes. J. Comput. Phys. 43, 357-372.

Rottman, J. W. \& Einaudi, F. 1993 Solitary waves in the atmosphere. J. Atmos. Sci. 50, 2116-2136.

Sснёr, C. \& Sмiтh, R. B. 1993 a shallow-water flow past an isolated topography. Part I: Vorticity production and wake formation. J. Atmos. Sci. 50, 1373-1400.

SchäR, C. \& Sмiтh, R. B. $1993 b$ shallow-water flow past an isolated topography. Part II: Transition to vortex shedding. J. Atmos. Sci. 50, 1401-1412.

Sмith, R. B. \& Sмiтh, D. F. 1995 Pseudo-inviscid wake formation by mountains in shallow-water flow with a drifting vortex. J. Atmos. Sci. 52, 436-454.

Whithaм, G. B. 1974 Linear and Nonlinear Waves. Wiley. 